This item was submitted to Loughborough's Research Repository by the author.

Items in Figshare are protected by copyright, with all rights reserved, unless otherwise indicated.

Spatial dynamics methods for solitary gravity-capillary water waves with an arbitrary distribution of vorticity

PLEASE CITE THE PUBLISHED VERSION

LICENCE

CC BY-NC-ND 4.0

REPOSITORY RECORD

Groves, Mark D., and E. Wahlen. 2019. "Spatial Dynamics Methods for Solitary Gravity-capillary Water Waves with an Arbitrary Distribution of Vorticity”. figshare. https://hdl.handle.net/2134/2401. 


\title{
Spatial dynamics methods for solitary gravity-capillary water waves with an arbitrary distribution of vorticity
}

\author{
M. D. Groves \\ Department of Mathematical Sciences \\ Loughborough University \\ Loughborough LE11 3TU \\ UK
}

\author{
E. Wahlén \\ Department of Mathematics \\ Lund University \\ 22100 Lund \\ Sweden
}

\begin{abstract}
This paper presents existence theories for several families of small-amplitude solitarywave solutions to the classical water-wave problem in the presence of surface tension and with an arbitrary distribution of vorticity. Moreover, the established local bifurcation diagram for irrotational solitary waves is shown to remain qualitatively unchanged for any choice of vorticity distribution.

The hydrodynamic problem is formulated as an infinite-dimensional Hamiltonian system in which the horizontal spatial direction is the time-like variable. A centre-manifold reduction technique is employed to reduce the system to a locally equivalent Hamiltonian system with a finite numer of degrees of freedom. Homoclinic solutions to the reduced system, which correspond to solitary water waves, are detected by a variety of dynamical systems methods.
\end{abstract}

\section{Introduction}

The gravity-capillary water-wave problem concerns the flow of a perfect fluid of unit density subject to the forces of gravity and surface tension; the fluid is bounded below by a rigid horizontal bottom $\{y=0\}$ and above by a free surface $\{y=\eta(x, t)\}$, where $\eta$ depends upon the horizontal spatial coordinate $x$ and time $t$. Travelling waves are waves which propagate from left to right with constant speed $c$ and without change of shape, so that $\eta(x, t)=\eta(x-c t)$. The two principal classes of travelling waves are Stokes waves, which are periodic in a frame of reference moving with the wave, and solitary waves, which have the property that $\eta(x-c t) \rightarrow 0$ as $x-c t \rightarrow \pm \infty$. In this paper we construct rigorous existence theories for solitary waves on flows with an arbitrary distribution of vorticity.

Working in a frame of reference moving with the wave, let us describe the velocity field $(u(x, y), v(x, y))$ within the fluid domain $D_{\eta}=\{(x, y): x \in \mathbb{R}, 0<y<\eta(x)\}$ in terms of a stream function $\psi(x, y)$ which satisfies $\psi_{x}=-v, \psi_{y}=u-c$ and suppose that $u<c$, so that $\psi_{y}<0$. The vortiticy $\omega(x, y)=v_{x}(x, y)-u_{y}(x, y)$ is known under this condition to be 
a function of the stream function $\psi$, and we specify its distribution by prescribing a vorticity function $\gamma$ such that $\omega=\gamma(\psi)$. The hydrodynamic problem is to solve the nonlinear elliptic equation

$$
\Delta \psi=-\gamma(\psi), \quad 0<y<\eta(x)
$$

subject to the Dirichlet boundary conditions

$$
\begin{aligned}
\psi(x, 0) & =0, \\
\psi(x, \eta(x)) & =m_{0},
\end{aligned}
$$

where $m_{0}<0$ is the constant mass flux, the asymptotic conditions

$$
\eta(x) \rightarrow d \quad \text { as } x \rightarrow \pm \infty
$$

and the nonlinear boundary condition

$$
\frac{1}{2}|\nabla \psi|^{2}+g(y-d)-\sigma\left[\frac{\eta_{x}}{\sqrt{1+\eta_{x}^{2}}}\right]_{x}=\frac{\lambda}{2} \quad \text { on } y=\eta(x),
$$

where $g, \sigma$ and $d$ are respectively the acceleration due to gravity, the coefficient of surface tension and the asymptotic depth of the water, and $\lambda$ is a constant called the Bernoulli constant (e.g. see Constantin \& Strauss [11]).

Several existence theories for irrotational gravity-capillary solitary waves (where $\gamma=0$ ) are available in the literature (see below), and many of them use spatial dynamics methods. The phrase 'spatial dynamics' refers to an approach where a system of partial differential equations governing a physical problem is formulated as a (typically ill-posed) evolutionary equation

$$
u_{\xi}=L(u)+N(u),
$$

in which an unbounded spatial coordinate plays the role of the time-like variable $\xi$. The waterwave problem has one bounded or semi-bounded coordinate, namely the vertical coordinate $y$; by contrast no restriction is placed upon the behaviour of the waves in the horizontal coordinate $x$, and so this coordinate qualifies as 'time-like'. One therefore studies the problem using spatial dynamics by formulating it as an evolutionary system of the form (6), where $\xi=x$, in an infinite-dimensional phase space consisting of functions of $y$. Notice that the hydrodynamic problem is conservative and isotropic in $x$, and these symmetries manifest themselves in the fact that its spatial dynamics formulation is Hamiltonian and reversible. In Section 2 we derive a formulation of the water-wave problem with an arbitrary choice of $\gamma \in L^{2}\left(m_{0}, 0\right)$ as a reversible Hamiltonian system and place it in a secure functional-analytic framework.

One particularly useful technique for finding solutions of (6) is known as centre-manifold reduction. Supposing that $L$ has a finite number of purely imaginary eigenvalues and that certain technical hypotheses are satisfied, one can show that (6) admits an invariant manifold called the centre manifold which contains all its small, bounded solutions; the dimension of the centre manifold is given by the number of purely imaginary eigenvalues. This reduction procedure is explained in detail by Mielke [32], and is shown in Section 3.1 to be applicable to our spatial 
dynamics formulation of the gravity-capillary water-wave problem for fluid of finite depth and with any choice of $\gamma \in H^{1}\left(m_{0}, 0\right)$ which satisfies

$$
\int_{0}^{1} \frac{1}{\sqrt{2 \Gamma(s)-2 \Gamma_{\min }}} \mathrm{d} s>1, \quad \Gamma_{\min }=\min _{s \in[0,1]} \Gamma(s),
$$

where

$$
\Gamma(s)=\frac{d^{2}}{m_{0}} \int_{s}^{1} \gamma\left(m_{0} u\right) \mathrm{d} u .
$$

(The additional regularity requirement on $\gamma$ simplifies the calculation of the spectrum of $L$.) An important aspect of the centre-manifold reduction procedure is that it preserves symmetries of the initial evolutionary equation. This feature can be exploited in existence theories for water waves: since our spatial dynamics formulation is Hamiltonian and reversible, the reduced system on the centre manifold is a reversible Hamiltonian system with finitely many degrees of freedom.

Bifurcation phenomena obtained by varying a parameter can also be captured by the centremanifold reduction procedure. A bifurcation parameter $\varepsilon$ may be introduced by perturbing physical parameters present in the problem (in the case of water waves $\alpha=g d^{3} / m_{0}^{2}$ and $\beta=\sigma d / m_{0}^{2}$ ) around fixed reference values, and the reduction procedure delivers an $\varepsilon$-dependent manifold which captures the small-amplitude dynamics for small values of this parameter; the manifold is a true centre manifold at criticality $(\varepsilon=0)$, so that its dimension is the number of purely imaginary eigenvalues of $L$ at $\varepsilon=0$. The reduction procedure is therefore especially helpful in detecting bifurcations which are associated with a change in the number of purely imaginary eigenvalues. In the case of irrotational waves there are four critical curves $C_{1}, \ldots, C_{4}$ in the $(\beta, \alpha)$ parameter plane at which the number of purely imaginary eigenvalues of $L$ changes (see Figure 1(a)), and an explicit parameterisation of each of these curves is available (Kirchgässner [29]). Section 3.2 contains spectral theory which shows that this 'bifurcation diagram' remains qualitatively unchanged for each choice of vorticity function $\gamma$; explicit formulae are however only available for $C_{3}=\left\{\left(\beta, \alpha_{\star}\right): \beta<\beta_{\star}\right\}$ and $C_{4}=\left\{\left(\beta, \alpha_{\star}\right): \beta>\beta_{\star}\right\}$, where $\beta_{\star}$, $\alpha_{\star}$ are positive constants determined by the choice of $\gamma$ (the values of $\beta_{\star}$ and $\alpha_{\star}$ for $\gamma=0$ are respectively $1 / 3$ and 1$)$.

In irrotational water-wave theory the curves $C_{1}, C_{2}$ and $C_{4}$ are associated with homoclinic bifurcation, where solutions of the reduced Hamiltonian system which are asymptotically zero bifurcate from the trivial solution; these solutions correspond to solitary water waves. Figure 1 illustrates the regions I, II and III adjacent to respectively $C_{4}, C_{1}$ and $C_{2}$ in which homoclinic solutions exist for $\gamma=0$, and in the present paper we show that the same is true any choice of $\gamma \in H^{1}\left(m_{0}, 0\right)$ satisfying (7). There are however physical differences in the corresponding solitary waves: a small-amplitude irrotational solitary wave is a perturbation of a uniform flow, while a solitary wave with $\gamma \neq 0$ 'rides' a laminar flow in which the velocity field is horizontal but depth-dependent.

Irrotational waves in region I were studied by Kirchgässner [29] (see also Amick \& Kirchgässner [1], Sachs [33] and Iooss \& Kirchgässner [24]). A Hamiltonian $0^{2}$-resonance takes place at $C_{4}$, that is two imaginary eigenvalues collide at the origin and become real as one crosses the curve from below. Kirchgässner showed that the flow on the two-dimensional centre manifold is controlled by the reversible, Hamiltonian equation

$$
u_{x x}=u+\frac{3}{2} u^{2}+O\left(\delta^{1 / 2}\right)
$$




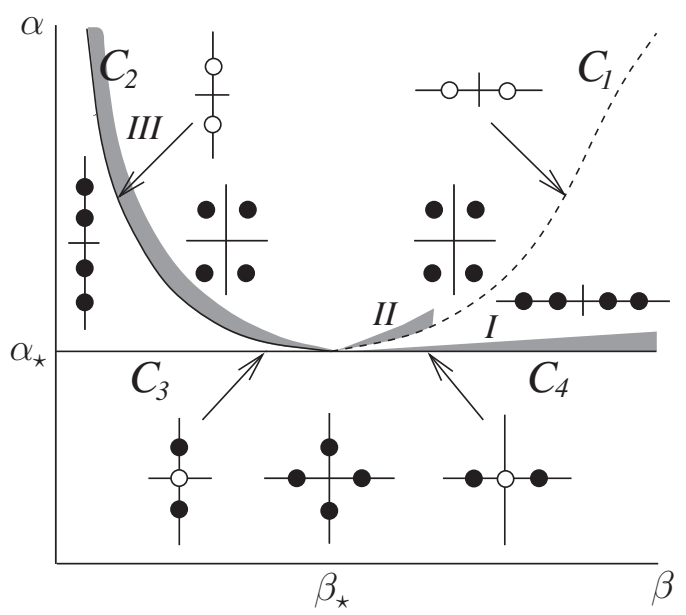

(a) Bifurcation curves in the $(\beta, \alpha)$-plane; the shaded regions indicate the parameter regimes in which homoclinic bifurcation is detected.

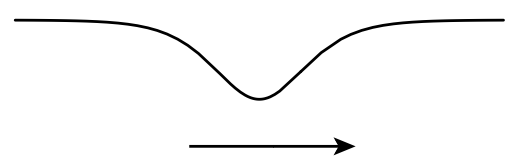

(b) A symmetric solitary wave of depression (left) is found in region I.
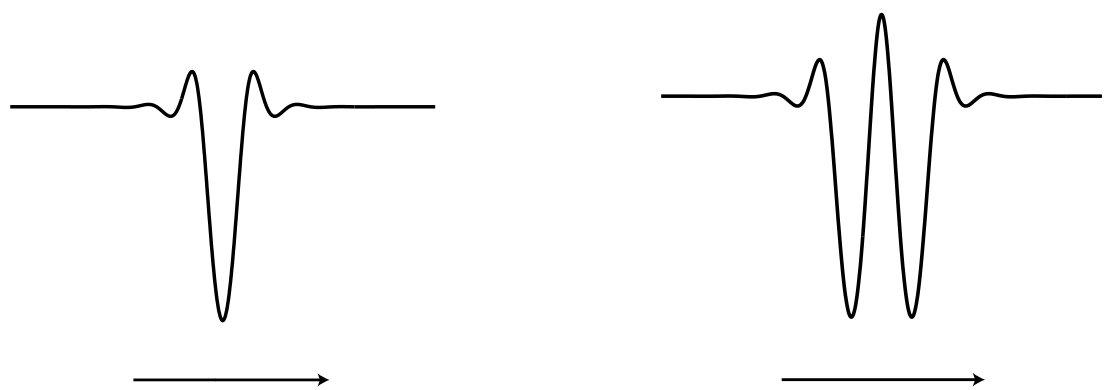

(c) Region II contains an infinite family of multi-troughed solitary waves which decay in an oscillatory fashion.
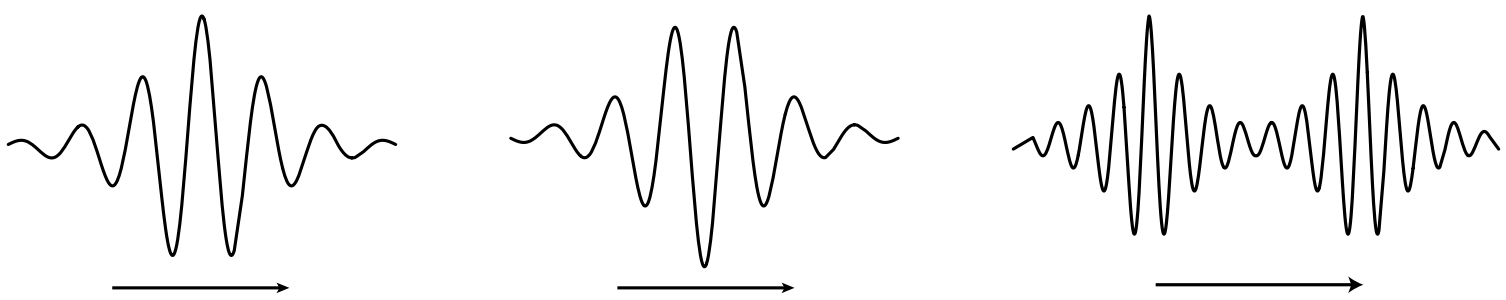

(d) Symmetric unipulse modulated solitary waves (left and centre) co-exist with an infinite family of multipulse modulated solitary waves (right) in region III.

Figure 1: Summary of the solitary waves whose existence is established in the present paper by centre-manifold reduction and homoclinic bifurcation theory. 
where $0<\delta \ll 1$ is the bifurcation parameter $\alpha-\alpha_{\star}$. This equation admits a homoclinic solution which corresponds to a solitary wave of depression whose tail decays exponentially and monotonically. In Section 4.1 we show that Kirchgässner's analysis and conclusions remain valid for an arbitary choice of $\gamma \neq 0$ and give a geometric interpretation of his method.

Region II lies on the 'complex side' of the curve $C_{1}$, at points of which two pairs of small-amplitude real eigenvalues collide and become complex. The centre manifold is fourdimensional, and it was shown by Buffoni, Groves \& Toland [8] that for $\gamma=0$ the flow on the centre manifold is controlled by the reversible, Hamiltonian equation

$$
u_{x x x x}-2(1+\delta) u_{x x}+u-u^{2}=0(\mu),
$$

where $0<\mu \ll 1$ measures the distance from the point $\left(\beta_{\star}, \alpha_{\star}\right)$ and $0<\delta \ll 1$ is the bifurcation parameter (measuring the distance from $C_{1}$ ). This equation has an infinite family of multipulse homoclinic solutions which make several large excursions away from the origin. The corresponding water waves are solitary waves of depression with $2,3,4, \ldots$ large troughs separated by $2,3, \ldots$ small oscillations; their tails are oscillatory and decay exponentially. In Section 4.2 we compute the reduced Hamiltonian system for a general choice of $\gamma$ using a method which is simpler than that employed by Buffoni, Groves \& Toland. We again arrive at (8), so that our hydrodynamic problem also admits a plethora of multipulse solitary waves in this parameter regime.

Region III was first examined by Iooss \& Kirchgässner [23], who studied homoclinic bifurcation associated with the Hamiltonian-Hopf bifurcation at points of $C_{2}$ (two pairs of purely imaginary eigenvalues collide at non-zero points $\pm \mathrm{i} q$ and become complex). The centre manifold is four-dimensional at Hamiltonian-Hopf points, and the two-degree-of-freedom reduced Hamiltonian system is conveniently studied using complex coordinates $(A, B)$ and a normalform transformation. Introducing a bifurcation parameter $\delta$ so that positive values of $\delta$ correspond to points on the 'complex' side $C_{2}$, one obtains the reduced Hamiltonian system

$$
\begin{gathered}
A_{x}=\frac{\partial H}{\partial \bar{B}}, \quad B_{x}=-\frac{\partial H}{\partial \bar{A}} \\
H=\mathrm{i} q(A \bar{B}-\bar{A} B)+|B|^{2}+H_{\mathrm{NF}}\left(|A|^{2}, \mathrm{i}(A \bar{B}-\bar{A} B), \delta\right)+O\left(|(A, B)|^{2}|(\delta, A, B)|^{n_{0}}\right),
\end{gathered}
$$

where $H_{\mathrm{NF}}$ is a real polynomial which satisfies $H_{\mathrm{NF}}(0,0, \delta)=0$; it contains the terms of order $3, \ldots, n_{0}+1$ in the Taylor expansion of $H$. Supposing that the coefficients of certain terms in $H_{\mathrm{NF}}$ have the correct sign, one finds that the 'truncated normal form' obtained by neglecting the remainder term admits a circle of homoclinic solutions, two of which persist when the remainder terms are reinstated (see Iooss \& Pérouème [26]). The corresponding water waves are symmetric solitary waves which take the form of periodic wave trains modulated by exponentially decaying envelopes. Buffoni \& Groves [7] strengthened this result by showing that the above Hamiltonian system in fact has an infinite number of geometrically distinct homoclinic solutions which generically resemble multiple copies of one of the homoclinic solutions found by Iooss \& Kirchgässner. The relevant normal-form coefficients were computed for irrotational waves by Buffoni \& Groves [7, Appendix B]. Although such explicit formulae are not available for a general choice of $\gamma$ it is possible to prove that the coefficients have the correct signs in the local part of region III near $\left(\beta_{\star}, \alpha_{\star}\right)$; this procedure is carried out in Section 4.3. 
A different homoclinic bifurcation phenomenon occurs at $C_{3}$, where a Hamiltonian $0^{2} \mathrm{i} \omega$ resonance takes place, that is two imaginary eigenvalues collide at the origin and become real as $C_{3}$ is crossed from above, while a second pair of eigenvalues remains on the imaginary axis. Lombardi [30] has proved the existence of irrotational generalised solitary waves in the region just below $C_{3}$; their pulse-like profile decays at infinity to a periodic ripple whose amplitude is exponentially small compared to that of the pulse. It is still an open question whether genuine solitary waves exist in this parameter regime, although Sun [34] has recently proved that they do not exist for values of $\beta$ close to $\beta_{\star}$ and there is strong numerical evidence that the same is true for all values of $\beta<\beta_{\star}$ (Champneys, Vanden-Broeck \& Lord [9]). The corresponding discussion for $\gamma \neq 0$ is beyond the scope of the present paper.

Spatial dynamics techniques are also used in a variety of existence proofs for other types of irrotational gravity-capillary water waves, notably in centre-manifold methods for three-dimensional travelling waves (Groves \& Mielke [18], Groves [15], Groves \& Haragus [16], Groves \& Sandstede [19]). On the other hand centre-manifold reduction is not available in other situations, for example in the existence theories for three-dimensional solitary waves by Groves, Haragus \& Sun [17] and for two-dimensional solitary waves on water of infinite depth by Iooss \& Kirrmann [25]; in these cases other methods are used to find solutions of the spatial dynamics formulation of the hydrodynamic problem as an inifinite-dimensional evolutionary equation.

The present contribution is one of a series of papers in which established existence theories for irrotational water waves are generalised to flows with arbitrary distributions of vorticity. This programme of research began with Constantin \& Strauss [11], who generalised Keady \& Norbury's [28] proof of the existence of a connected global branch of irrotational symmetric gravity Stokes waves containing waves whose speeds at the crest are arbitrarily small. Wahlén [35] has recently generalised the bifurcation theory for small-amplitude gravity-capillary Stokes waves by Jones [27] (see also Zeidler [36, 37]), while Hur [21] has extended Beale's [2] construction of small-amplitude gravity solitary waves.

\section{Formulation as a Hamiltonian system}

In this section we formulate the hydrodynamic problem as a reversible Hamiltonian system, the irrotational version of which was outlined by Groves [14] (see also Benjamin [3, Appendix B]). Note that the irrotational version differs from the Hamiltonian system due to Groves \& Toland [20] which is employed in the centre-manifold reduction methods for irrotational water waves described above.

We begin by writing the hydrodynamic problem (1)-(5) in terms of the dimensionless variables

$$
\left(x^{\prime}, y^{\prime}\right)=\frac{1}{d}(x, y), \quad \eta^{\prime}\left(x^{\prime}\right)=\frac{1}{d} \eta(x), \quad \psi^{\prime}\left(x^{\prime}, y^{\prime}\right)=-\frac{1}{m_{0}} \psi(x, y)
$$

and dimensionless vorticity function

$$
\gamma^{\prime}\left(\psi^{\prime}\right)=-\frac{d^{2}}{m_{0}} \gamma(\psi)
$$

One finds that

$$
\Delta \psi=-\gamma(\psi), \quad 0<y<\eta(x),
$$




$$
\begin{array}{rlr}
\psi(x, 0) & =0, & \\
\psi(x, \eta(x)) & =-1, & \\
\eta(x) & \rightarrow 1 & \text { as } x \rightarrow \pm \infty
\end{array}
$$

and

$$
\frac{1}{2}|\nabla \psi|^{2}+\alpha(y-1)-\beta\left[\frac{\eta_{x}}{\sqrt{1+\eta_{x}^{2}}}\right]_{x}=\frac{\mu}{2} \quad \text { on } y=\eta(x),
$$

in which

$$
\alpha=\frac{g d^{3}}{m_{0}^{2}}, \quad \beta=\frac{\sigma d}{m_{0}^{2}}, \quad \mu=\frac{\lambda d^{2}}{m_{0}^{2}}
$$

are dimensionless parameters and the primes have been dropped for notational simplicity. The next step is to map the unknown fluid domain $D_{\eta}$ into a fixed strip $\mathbb{R} \times(0,1)$ using a transformation devised by Dubreil-Jacotin [13]. We define $s=-\psi(x, y), h=y$, and treat $(x, s) \in$ $\mathbb{R} \times(0,1)$ as independent variables and $\eta(x), h(x, s)$ as dependent variables. A straightfoward calculation shows that equations (9)-(13) are transformed into

$$
\begin{array}{ll}
{\left[\frac{h_{x}}{h_{s}}\right]_{x}-\left[\frac{1+h_{x}^{2}}{2 h_{s}^{2}}\right]_{s}+\gamma(-s)=0,} & 0<s<1, \\
h(x, 0)=0, & \\
h(x, 1)=\eta, & \text { as } x \rightarrow \pm \infty \\
\eta(x) \rightarrow 1 & s=1, \\
\frac{1+h_{x}^{2}}{2 h_{s}^{2}}+\alpha(h-1)-\beta\left[\frac{h_{x}}{\sqrt{1+h_{x}^{2}}}\right]_{x}=\frac{\mu}{2}, & s=1,
\end{array}
$$

and we seek solutions with $h_{s}>0$, a condition which is implied by the assumption $\psi_{y}<0$ (Constantin \& Strauss [11]). The following proposition, which is proved by straightforward arguments from the theory of elliptic boundary-value problems, relates solutions of the transformed equations to those of (9)-(13).

Proposition 2.1 Define $I=\left(x_{1}, x_{2}\right), I^{\prime}=\left(x_{1}^{\prime}, x_{2}^{\prime}\right)$ with $x_{1}<x_{1}^{\prime}<x_{2}^{\prime}<x_{2}$ and let $D_{\eta, I}=$ $\{(x, y): x \in I, 0<y<\eta(x)\}$.

(i) Suppose that $\gamma \in L^{2}(-1,0)$. Any solution $h \in H^{2}(I \times(0,1)) \cap C^{1}(I \times[0,1])$ and $\eta=\left.h\right|_{\{s=1\}} \in C^{2}(I)$ of (14)-(16), (18) defines a solution $\psi \in H^{2}\left(D_{\eta, I^{\prime}}\right) \cap C^{1}\left(\overline{D_{\eta, I^{\prime}}}\right)$, $\eta \in C^{2}(I)$ of (9)-(11), (13).

(ii) The additional regularity $\gamma \in C^{k, \alpha}[-1,0]$ and $\eta \in C^{k+2, \alpha}(I)$ for some $\alpha \in(0,1)$ and some nonnegative integer $k$ implies that $\psi \in C^{k+2, \alpha}\left(\overline{D_{\eta, I^{\prime}}}\right)$.

Observe that equations (14)-(16), (18) follow from the formal variational principle

$$
\delta \mathcal{J}=0
$$

where

$$
\mathcal{J}=\int\left\{\int_{0}^{1}\left(-\frac{1+h_{x}^{2}}{2 h_{s}^{2}}+\alpha(h-1)-\frac{\mu}{2}-\Gamma(s)\right) h_{s} \mathrm{~d} s+\beta \sqrt{1+\eta_{x}^{2}}\right\} \mathrm{d} x
$$




$$
\Gamma(s)=-\int_{s}^{1} \gamma(-u) d u, \quad s \in[0,1]
$$

and the variations are taken with respect to $\eta(x)$ and $h(x, s)$ such that $h(x, 1)=\eta(x)$ and $h(x, 0)=0$. (The corresponding variational principle for gravity waves was given by Constantin, Sattinger \& Strauss [10].) We exploit this variational principle by regarding $\mathcal{L}$ as an action functional of the form

$$
\mathcal{J}=\int J\left(\eta, h, \eta_{x}, h_{x}\right) \mathrm{d} x
$$

and deriving a Hamiltonian formulation of (14)-(16), (18) by means of the Legendre transform. To this end, let us introduce new variables $\omega$ and $w$ by the formulae

$$
\omega=\frac{\delta \mathcal{J}}{\delta \eta_{x}}=\frac{\beta \eta_{x}}{\sqrt{1+\eta_{x}^{2}}}, \quad w=\frac{\delta \mathcal{J}}{\delta h_{x}}=-\frac{h_{x}}{h_{s}},
$$

in which the variational derivatives are taken in respectively $L^{2}(\mathbb{R})$ and $L^{2}(\mathbb{R} \times(0,1))$, and define the Hamiltonian function by

$$
\begin{aligned}
H(\eta, \omega, h, w) & \\
\quad= & \int_{0}^{1} w h_{x} \mathrm{~d} s+\omega \eta_{x}-J\left(\eta, h, \eta_{x}, h_{x}\right) \\
& =\int_{0}^{1}\left\{\frac{1}{2}\left(\frac{1}{h_{s}}-h_{s} w^{2}\right)+\Gamma(s) h_{s}\right\} \mathrm{d} s-\frac{1}{2} \alpha(\eta-1)^{2}+\frac{1}{2} \alpha+\frac{\mu}{2} \eta-\sqrt{\beta^{2}-\omega^{2}} .
\end{aligned}
$$

This procedure suggests that the equations

$$
\eta_{x}=\frac{\delta H}{\delta \omega}, \quad \omega_{x}=-\frac{\delta H}{\delta \eta}, \quad h_{x}=\frac{\delta H}{\delta w}, \quad w_{x}=-\frac{\delta H}{\delta h}
$$

formally represent Hamilton's equations for a formulation of the hydrodynamic problem (14)(16), (18) as a Hamiltonian system.

In order to make the above suggestion rigorous, we define the Hilbert spaces

$$
\begin{aligned}
& X=\left\{(\eta, \omega, h, w) \in \mathbb{R} \times \mathbb{R} \times H^{1}(0,1) \times L^{2}(0,1): h(0)=0, h(1)=\eta\right\}, \\
& Y=\left\{(\eta, \omega, h, w) \in \mathbb{R} \times \mathbb{R} \times H^{2}(0,1) \times H^{1}(0,1): h(0)=0, h(1)=\eta\right\}
\end{aligned}
$$

and consider the symplectic manifold $(X, \Omega)$, where $\Omega$ is the position-independent 2 -form on $X$ given by

$$
\left.\Omega\right|_{(\eta, \omega, h, w)}\left(\left(\eta_{1}, \omega_{1}, h_{1}, w_{1}\right),\left(\eta_{2}, \omega_{2}, h_{2}, w_{2}\right)\right)=\int_{0}^{1}\left(w_{2} h_{1}-w_{1} h_{2}\right) \mathrm{d} s+\omega_{2} \eta_{1}-\omega_{1} \eta_{2}
$$

(the canonical 2-form with respect to the $\mathbb{R} \times \mathbb{R} \times L^{2}(0,1) \times L^{2}(0,1)$-inner product). Choose $\gamma \in L^{2}(-1,0)$, so that $\Gamma \in H^{1}(-1,0)$, and observe that the set

$$
M=\left\{(\eta, \omega, h, w) \in Y:|\omega|<\beta, \eta>0, h_{s}(s)>0 \text { for each } s \in[0,1]\right\}
$$


is a manifold domain of $X$ and that the function $H$ given by (20) belongs to $C^{\infty}(M, \mathbb{R})$; a direct calculation shows that

$$
\begin{aligned}
\left.\mathrm{d} H\right|_{m}\left(\left.v_{1}\right|_{m}\right)=\int_{0}^{1}\{- & \left.\frac{1}{2}\left(w^{2}+\frac{1}{h_{s}^{2}}\right)+\Gamma(p)\right\} h_{1 s} \mathrm{~d} s-\int_{0}^{1} h_{s} w w_{1} \mathrm{~d} s \\
& -\alpha(\eta-1) \eta_{1}+\frac{\mu}{2} \eta_{1}+\frac{\omega \omega_{1}}{\sqrt{\beta^{2}-\omega^{2}}}
\end{aligned}
$$

for $m=(\eta, \omega, h, w) \in M$ and $\left.v_{1}\right|_{m}=\left.\left(\eta_{1}, \omega_{1}, h_{1}, w_{1}\right) \in T M\right|_{m} \cong Y$. The triple $(X, \Omega, H)$ is therefore a Hamiltonian system.

Recall that the point $m \in M$ belongs to $\mathcal{D}\left(v_{H}\right)$ with $\left.v_{H}\right|_{m}=\left.\bar{v}\right|_{m}$ if and only if

$$
\Omega_{m}\left(\left.\bar{v}\right|_{m},\left.v_{1}\right|_{m}\right)=\left.\mathrm{d} H\right|_{m}\left(\left.v_{1}\right|_{m}\right)
$$

for all tangent vectors $\left.\left.v_{1}\right|_{m} \in T X\right|_{m}$ (note that $\left.\left.\mathrm{d} H\right|_{m} \in T^{*} M\right|_{m} \cong Y^{*}$ extends to an element of $\left.T^{*} X\right|_{m} \cong X^{*}$ because $Y$ is dense in $X$ ). Using this criterion, the above expression for $\left.\mathrm{d} H\right|_{m}\left(\left.v_{1}\right|_{m}\right)$ and integrating by parts, one finds that

$$
\mathcal{D}\left(v_{H}\right)=\left\{(\eta, \omega, h, w) \in M: w(0)=0, h_{s}(1) w(1)=-\frac{\omega}{\sqrt{\beta^{2}-\omega^{2}}}\right\}
$$

and that Hamilton's equations

$$
u_{x}=v_{H}(u)
$$

are given explicitly by

$$
\begin{aligned}
\eta_{x} & =\frac{\omega}{\sqrt{\beta^{2}-\omega^{2}}}, \\
\omega_{x} & =\frac{1}{2}\left(w^{2}(1)+\frac{1}{h_{s}^{2}(1)}\right)+\alpha(\eta-1)-\frac{\mu}{2} \\
h_{x} & =-h_{s} w \\
w_{x} & =-\frac{1}{2}\left(w^{2}+\frac{1}{h_{s}^{2}}\right)_{s}+\gamma(-s) .
\end{aligned}
$$

Observe that Hamilton's equations are reversible; the reverser $S: X \rightarrow X$ is defined by $S(\eta, \omega, h, w)=(\eta,-\omega, h,-w)$.

Proposition 2.2 Suppose that $(\eta, \omega, h, w) \in C\left(I, \mathcal{D}\left(v_{H}\right)\right) \cap C^{1}(I, X), I=\left(x_{1}, x_{2}\right)$ solves Hamilton's equations and let $I^{\prime}=\left(x_{1}^{\prime}, x_{2}^{\prime}\right)$ with $x_{1}<x_{1}^{\prime}<x_{2}^{\prime}<x_{2}$. The functions $\tilde{h}, \tilde{w}$ defined by

$$
\tilde{h}(x, s)=h(x)(s), \quad \tilde{w}(x, s)=w(x)(s)
$$

belong to respectively $H^{2}\left(D_{\eta, I^{\prime}}\right) \cap C^{1}\left(\overline{D_{\eta, I^{\prime}}}\right)$ and $H^{1}\left(D_{\eta, I^{\prime}}\right) \cap C\left(\overline{D_{\eta, I^{\prime}}}\right)$, while $\eta$ and $\omega$ belong to respectively $C^{2}\left(\overline{I^{\prime}}\right)$ and $C^{1}\left(\overline{I^{\prime}}\right)$. These functions satisfy $\tilde{h}(x, s)>0$ in $\overline{D_{\eta, I^{\prime}}},|\omega|<\beta$ in $\overline{I^{\prime}}$, the equations

$$
\tilde{h}_{x}=-\tilde{h}_{s} \tilde{w}, \quad \tilde{w}_{x}=-\frac{1}{2}\left(\tilde{w}^{2}-\frac{1}{\tilde{h}_{s}^{2}}\right)_{s}+\gamma(-s)
$$


in $D_{\eta, I^{\prime}}$ with boundary conditions

$$
\tilde{h}(x, 0)=\tilde{w}(x, 0)=0, \quad \tilde{h}(x, 1)=\eta(x), \quad \tilde{h}_{s}(x, 1) \tilde{w}(x, 1)=-\frac{\omega}{\sqrt{\beta^{2}-\omega^{2}}}
$$

and the equations

$$
\begin{aligned}
\eta_{x} & =\frac{\omega}{\sqrt{\beta^{2}-\omega^{2}}}, \\
\omega_{x} & =\left.\frac{1}{2}\left(\tilde{w}^{2}+\frac{1}{\tilde{h}_{s}^{2}}\right)\right|_{s=1}+\alpha(\eta-1)-\frac{\mu}{2}
\end{aligned}
$$

in $I^{\prime}$.

The above proposition is proved using the methods given by Groves \& Toland [20]. Eliminating $\omega$ and $\tilde{w}$ between the above equations, we find that $\tilde{h}$ and $\eta$ satisfy equations (14)-(16), (18) and Proposition 2.1 yields a solution of the hydrodynamic problem (9)-(11), (13). Note that the additional regularity $\gamma \in C^{k, \alpha}[0,1]$ and $u \in C^{k+2}\left(I, \mathcal{D}\left(v_{H}\right)\right) \cap C^{k+3}(I, X)$ for some $\alpha \in(0,1)$ and some nonnegative integer $k$ implies that $\psi \in C^{k+2, \alpha}\left(\overline{D_{\eta, I^{\prime}}}\right)$. In the remainder of this article we take $\gamma \in H^{1}(0,1)$ rather than $\gamma \in L^{2}(0,1)$ in order to simplify the spectral theory presented in Section 3.2.

We proceed by seeking solutions $(\eta, \omega, h, w) \in C\left(\mathbb{R}, \mathcal{D}\left(v_{H}\right)\right) \cap C^{1}(\mathbb{R}, X)$ of Hamilton's equations which satisfy $\eta(x) \rightarrow 1$ as $x \rightarrow \pm \infty$. These solutions take the form of perturbations of equilibrium (that is $x$ independent) solutions $\left(\eta_{0}, \omega_{0}, h_{0}(s), w_{0}(s)\right)$, where necessarily $\eta_{0}=1$ and $\omega_{0}=0, w_{0}=0$ (see equation (19)); our solitary waves therefore 'ride' a horizontal laminar flow (which is in general not uniform). The requirement that the hydrodynamic problem admits a horizontal laminar flow for a given vorticity function fixes the value of the Bernoulli constant $\mu$.

\section{Lemma 2.3 Suppose that}

$$
\int_{0}^{1} \frac{1}{\sqrt{2 \Gamma(s)-2 \Gamma_{\min }}} \mathrm{d} s>1
$$

where

$$
\Gamma_{\min }=\min _{s \in[0,1]} \Gamma(s), \quad \Gamma_{\max }=\max _{s \in[0,1]} \Gamma(s) .
$$

There exists a unique value $\mu^{\star}>-2 \Gamma_{\min }$ of $\mu$ for which Hamilton's equations (21)-(24) admit a solution of the form

$$
(\eta, \omega, h, w)=(1,0, \theta(s), 0)
$$

for all $\beta, \alpha>0$. The function $\theta(s)$ is given by the formula

$$
\theta(s)=\int_{0}^{s} a^{-1}(u) \mathrm{d} u, \quad a(s)=\sqrt{\mu^{\star}+2 \Gamma(s)} .
$$


Proof. Any solution of (21)-(24) of the form (26) satisfies

$$
\frac{1}{2}\left(\frac{1}{\theta_{s}^{2}}\right)_{s}=\gamma(-s), \quad \frac{1}{\theta_{s}^{2}(1)}=\mu, \quad \theta(0)=0,
$$

so that

$$
\theta(s)=\int_{0}^{s} \frac{1}{\sqrt{\mu+2 \Gamma(u)}} \mathrm{d} u,
$$

where we have assumed that $\mu>-2 \Gamma_{\min }$. It remains to satisfy the boundary condition $h(1)=\eta$, that is $\theta(1)=1$. Observe that $\theta(1)$ is a strictly decreasing function of $\mu \in\left[-2 \Gamma_{\min }, \infty\right)$ which satisfies $\theta(1) \rightarrow 0$ as $\mu \rightarrow \infty$ and $\left.\theta(1)\right|_{\mu=-2 \Gamma_{\min }}>1$ under the assumption (25). It follows that there is a unique value $\mu^{\star}$ of $\mu$ such that $\theta(1)=1$.

In accordance with Lemma 2.3 we take $\mu=\mu^{\star}$ and seek solutions of Hamilton's equations for $(X, \Omega, H)$ of the form

$$
\eta=1+\rho, \quad h=\theta+\phi,
$$

where $\rho>-1$ and $\phi(s)>-a^{-1}(s)$ for $s \in[0,1]$. Let us write

$$
(\beta, \alpha)=\left(\beta_{0}+\varepsilon_{1}, \alpha_{0}+\varepsilon_{2}\right),
$$

where $\left(\beta_{0}, \alpha_{0}\right)$ is fixed and $\left(\varepsilon_{1}, \varepsilon_{2}\right)$ lies in a neighbourhood $\Lambda$ of the origin in $\mathbb{R}^{2}$, and consider solutions $(\rho, \omega, \phi, w)$ which lie in a neighbourhood $V$ of the origin in $Y$; here $\Lambda$ and $V$ are chosen small enough so that

$\left|\varepsilon_{1}\right|<\frac{\beta_{0}}{4}, \quad \rho>-\frac{1}{2}>-1, \quad|\omega|<\frac{\beta_{0}}{2}<\beta_{0}+\varepsilon_{1}, \quad \phi_{s}(s)>-\frac{1}{2}\left(\mu^{\star}+2 \Gamma_{\max }\right)^{-1 / 2}>-a^{-1}(s)$

for each $s \in[0,1]$. This change of variable transforms $(X, \Omega, H)$ into $\left(X, \Omega, H^{\varepsilon}\right)$, where $H^{\varepsilon} \in$ $C^{\infty}(V, \mathbb{R})$ is defined by the formula

$$
\begin{aligned}
H^{\varepsilon}(\rho, \omega, \phi, w)=\int_{0}^{1}\{ & \left.\frac{1}{2}\left(\frac{1-\left(a^{-1}(s)+\phi_{s}\right)^{2} w^{2}}{a^{-1}(s)+\phi_{s}}\right)-\frac{1}{2} a(s)+\Gamma(s) \phi_{s}\right\} \mathrm{d} s \\
& -\frac{1}{2}\left(\alpha_{0}+\varepsilon_{2}\right)^{2} \rho^{2}+\frac{1}{2} \mu^{\star} \rho+\left(\beta_{0}+\varepsilon_{1}\right)-\sqrt{\left(\beta_{0}+\varepsilon_{1}\right)^{2}-\omega^{2}}
\end{aligned}
$$

(a constant term has also been added to the Hamiltonian to ensure that $H^{\varepsilon}(0)=0$ ). Hamilton's equations (21)-(24) are transformed into

$$
\begin{aligned}
\rho_{x} & =\frac{\omega}{\sqrt{\left(\beta_{0}+\varepsilon_{1}\right)^{2}-\omega^{2}}}, \\
\omega_{x} & =\frac{1}{2}\left(w^{2}(1)+\frac{a^{2}(1)}{\left(1+a(1) \phi_{s}(1)\right)^{2}}\right)+\left(\alpha_{0}+\varepsilon_{2}\right) \rho-\frac{\mu^{\star}}{2}, \\
\phi_{x} & =-\left(a^{-1}(s)+\phi_{s}\right) w \\
w_{x} & =-\frac{1}{2}\left(w^{2}+\frac{a^{2}(s)}{\left(1+a(s) \phi_{s}\right)^{2}}\right)_{s}+\gamma(-s),
\end{aligned}
$$


the domain $\mathcal{D}\left(v_{H^{\varepsilon}}\right)$ of the Hamiltonian vector field on the right-hand side of this system of equations is the set of elements $(\rho, \omega, \phi, w) \in V$ which satisfy

$$
\begin{aligned}
w(0) & =0, \\
\left(\phi_{s}(1)+a^{-1}(1)\right) w(1) & =-\frac{\omega}{\sqrt{\left(\beta+\varepsilon_{1}\right)^{2}-\omega^{2}}}
\end{aligned}
$$

and the action of the reverser $S: X \rightarrow X$ is given by $S(\rho, \omega, \phi, w)=(\rho,-\omega, \phi,-w)$. Our task is to find homoclinic solutions of the above equations, that is solutions $(\rho, \omega, \phi, w) \in$ $C\left(\mathbb{R}, \mathcal{D}\left(v_{H}^{\varepsilon}\right)\right) \cap C^{1}(\mathbb{R}, X)$ which satisfy $(\rho(x), \omega(x), \phi(x), w(x)) \rightarrow(0,0,0,0)$ as $x \rightarrow \pm \infty$.

\section{Centre-manifold reduction}

\subsection{Application of the reduction theorem}

Our strategy in finding solutions to Hamilton's equations (27)-(30) for $\left(X, \Omega, H^{\varepsilon}\right)$ consists in applying a reduction principle which asserts that $\left(X, \Omega, H^{\varepsilon}\right)$ is locally equivalent to a finitedimensional Hamiltonian system. The key result is the following theorem, which is a parameterised, Hamiltonian version of a reduction principle for quasilinear evolutionary equations presented by Mielke [31, Theorem 4.1] (see Buffoni, Groves \& Toland [8, Theorem 4.1]).

Theorem 3.1 Consider the differential equation

$$
u_{x}=\mathcal{L} u+\mathcal{N}(u ; \lambda),
$$

which represents Hamilton's equations for the reversible Hamiltonian system $\left(X, \Omega^{\lambda}, H^{\lambda}\right)$. Here $u$ belongs to a Hilbert space $\mathcal{X}, \lambda \in \mathbb{R}^{\ell}$ is a parameter and $\mathcal{L}: \mathcal{D}(\mathcal{L}) \subset \mathcal{X} \rightarrow \mathcal{X}$ is a densely defined, closed linear operator. Regarding $\mathcal{D}(\mathcal{L})$ as a Hilbert space equipped with the graph norm, suppose that 0 is an equilibrium point of (32) when $\lambda=0$ and that

(H1) The part of the spectrum $\sigma(\mathcal{L})$ of $\mathcal{L}$ which lies on the imaginary axis consists of a finite number of eigenvalues of finite multiplicity and is separated from the rest of $\sigma(\mathcal{L})$ in the sense of Kato, so that $\mathcal{X}$ admits the decomposition $\mathcal{X}=\mathcal{X}_{1} \oplus \mathcal{X}_{2}$, where $\mathcal{X}_{1}=\mathcal{P}(\mathcal{X})$, $\mathcal{X}_{2}=(I-\mathcal{P})(\mathcal{X})$ and $\mathcal{P}$ is the spectral projection corresponding the purely imaginary part of $\sigma(\mathcal{L})$.

(H2) The operator $\mathcal{L}_{2}=\left.\mathcal{L}\right|_{\mathcal{X}_{2}}$ satisfies the estimate

$$
\left\|\left(\mathcal{L}_{2}-\mathrm{i} \xi I\right)^{-1}\right\|_{\mathcal{X}_{2} \rightarrow \mathcal{X}_{2}} \leq \frac{C}{1+|\xi|}, \quad \xi \in \mathbb{R}
$$

for some constant $C$ that is independent of $\xi$.

(H3) There exists a natural number $k$ and neighbourhoods $\Lambda \subset \mathbb{R}^{\ell}$ of 0 and $U \subset \mathcal{D}(\mathcal{L})$ of 0 such that $\mathcal{N}$ is $(k+1)$ times continuously differentiable on $U \times \Lambda$, its derivatives are bounded and uniformly continuous on $U \times \Lambda$ and $\mathcal{N}(0,0)=0, \mathrm{~d}_{1} \mathcal{N}[0,0]=0$. 
Under these hypotheses there exist neighbourhoods $\tilde{\Lambda} \subset \Lambda$ of 0 and $\tilde{U}_{1} \subset U \cap \mathcal{X}_{1}, \tilde{U}_{2} \subset U \cap \mathcal{X}_{2}$ of 0 and a reduction function $r: \tilde{U}_{1} \times \tilde{\Lambda} \rightarrow \tilde{U}_{2}$ with the following properties. The reduction function $r$ is $k$ times continuously differentiable on $\tilde{U}_{1} \times \tilde{\Lambda}$, its derivatives are bounded and uniformly continuous on $\tilde{U}_{1} \times \tilde{\Lambda}$ and $r(0 ; 0)=0, \mathrm{~d}_{1} r[0 ; 0]=0$. The graph $\tilde{X}^{\lambda}=\left\{u_{1}+r\left(u_{1} ; \lambda\right) \in\right.$ $\left.\tilde{U}_{1} \times \tilde{U}_{2}: u_{1} \in \tilde{U}_{1}\right\}$ is a Hamiltonian centre manifold for (32), so that

(i) $\tilde{X}^{\lambda}$ is a locally invariant manifold of (32): through every point in $\tilde{X}^{\lambda}$ there passes a unique solution of (32) that remains on $\tilde{X}^{\lambda}$ as long as it remains in $\tilde{U}_{1} \times \tilde{U}_{2}$.

(ii) Every small bounded solution $u(x), x \in \mathbb{R}$ of (32) that satisfies $\left(u_{1}(x), u_{2}(x)\right) \in \tilde{U}_{1} \times$ $\tilde{U}_{2}$ lies completely in $\tilde{X}^{\lambda}$.

(iii) Every solution $u_{1}:\left(x_{1}, x_{2}\right) \rightarrow \tilde{U}_{1}$ of the reduced equation

$$
u_{1 x}=\mathcal{L} u_{1}+\mathcal{P} \mathcal{N}\left(u_{1}+r\left(u_{1} ; \lambda\right) ; \lambda\right)
$$

generates a solution

$$
u(x)=u_{1}(x)+r\left(u_{1}(x) ; \lambda\right)
$$

of the full equation (32).

(iv) $\tilde{X}^{\lambda}$ is a symplectic submanifold of $X$ and the flow determined by the Hamiltonian system $\left(\tilde{X}^{\lambda}, \tilde{\Omega}^{\lambda}, \tilde{H}^{\lambda}\right)$, where the tilde denotes restriction to $\tilde{X}^{\lambda}$, coincides with the flow on $\tilde{X}^{\lambda}$ determined by $\left(X, \Omega^{\lambda}, H^{\lambda}\right)$. The reduced equation (33) is reversible and represents Hamilton's equations for $\left(\tilde{X}^{\lambda}, \tilde{\Omega}^{\lambda}, \tilde{H}^{\lambda}\right)$.

The centre manifold $\tilde{X}^{\lambda}$ is equipped with the single coordinate chart $\tilde{U}_{1} \subset \mathcal{X}_{1}$ and coordinate map $\chi: \tilde{X}^{\lambda} \rightarrow \tilde{U}_{1}$ defined by $\chi^{-1}\left(u_{1}\right)=u_{1}+r\left(u_{1} ; \lambda\right)$. It is however more convenient to use an alternative coordinate map for calculations. According to the parameter-dependent version of Darboux's theorem presented by Buffoni \& Groves [7, Theorem 4] there exists a near-identity change of variable

$$
\tilde{u}_{1}=u_{1}+\Theta\left(u_{1} ; \lambda\right)
$$

of class $C^{k-1}$ which transforms $\tilde{\Omega}^{\lambda}$ into $\Psi$, where

$$
\Psi\left(v_{1}, v_{2}\right)=\left.\Omega^{0}\right|_{0}\left(v_{1}, v_{2}\right) .
$$

Define the function $\tilde{r}: \tilde{U}_{1} \times \tilde{\Lambda} \rightarrow \tilde{U}_{1} \times \tilde{U}_{2}$ (which in general has components in $\mathcal{X}_{1}$ and $\mathcal{X}_{2}$ ) by the formula

$$
\tilde{u}_{1}+\tilde{r}\left(\tilde{u}_{1} ; \lambda\right)=\mathcal{T}\left(u_{1}+r\left(u_{1} ; \lambda\right) ; \lambda\right), \quad \mathcal{T}\left(u_{1}, u_{2} ; \lambda\right)=\left(u_{1}+\Theta\left(u_{1} ; \lambda\right), u_{2}\right),
$$

where $\tilde{r}(0 ; 0)=0, \mathrm{~d}_{1} \tilde{r}[0 ; 0]=0$, and equip $\tilde{X}^{\lambda}$ with the coordinate map $\tilde{\chi}: \tilde{X}^{\lambda} \rightarrow \tilde{U}_{1}$ given by $\tilde{\chi}^{-1}\left(\tilde{u}_{1}\right)=\tilde{u}_{1}+\tilde{r}\left(\tilde{u}_{1} ; \lambda\right)$. One can always choose a basis for $\mathcal{X}_{1}$ so that $\Psi$ is the canonical symplectic 2 -form $\Upsilon$ in this coordinate system (a 'symplectic basis'). The choice of $\tilde{\chi}$ as coordinate map therefore yields a finite-dimensional canonical Hamiltonian system. 
Theorem 3.1 cannot be applied directly to equations (27)-(28) because of the nonlinear boundary condition (31). We overcome this difficulty by using the change of variable $(\rho, \zeta, \phi, z)=$ $G^{\varepsilon}(\rho, \omega, \phi, w)$, where

$$
\zeta=-\frac{a(1) \omega}{\sqrt{\left(\beta+\varepsilon_{1}\right)^{2}-\omega^{2}}}, \quad z(s)=\left(1+a(s) \phi_{s}(s)\right) w(s),
$$

which transforms the nonlinear boundary condition into the linear condition $z(1)=\zeta$. The following lemma shows that $G^{\varepsilon}$ defines a valid change of variable.

\section{Lemma 3.2}

(i) For each $\varepsilon \in \Lambda$ the mapping $G^{\varepsilon}$ is a smooth diffeomorphism from the neighbourhood $V$ of 0 in $Y$ onto itself.

(ii) For each $(v, \varepsilon) \in V \times \Lambda$ the operators $\mathrm{d} G^{\varepsilon}[v], \mathrm{d}\left(\left(G^{\varepsilon}\right)^{-1}\right)[v]: Y \rightarrow Y$ extend to diffeomorphisms $X \rightarrow X$ that depend smoothly upon $(v, \varepsilon) \in V \times \Lambda$.

Proof. These results follow from the explicit formulae

$$
G^{-1}(\rho, \zeta, \phi, z)=(\rho, \omega, \phi, w)
$$

where

$$
\omega=-\frac{\left(\beta_{0}+\varepsilon_{1}\right) \zeta}{\sqrt{\zeta^{2}+a^{2}(1)}}, \quad w(s)=\frac{z(s)}{1+a(s) \phi_{s}(s)}
$$

and

$$
\begin{aligned}
& \mathrm{d} G^{\varepsilon}[\rho, \omega, \phi, w]\left(\rho_{1}, \omega_{1}, \phi_{1}, w_{1}\right) \\
&=\left(\rho_{1},-\frac{a(1)\left(\beta_{0}+\varepsilon_{1}\right)^{2} \omega_{1}}{\left(\left(\beta_{0}+\varepsilon_{1}\right)^{2}-\omega^{2}\right)^{3 / 2}}, \phi_{1},\left(1+a(s) \phi_{s}\right) w_{1}+a(s) w \phi_{1 s}\right) \\
&\left.\mathrm{d}\left(\left(G^{\varepsilon}\right)^{-1}\right)\right)[\rho, \zeta, \phi, z]\left(\rho_{1}, \zeta_{1}, \phi_{1}, z_{1}\right) \\
&=\left(\rho_{1},-\frac{\left(\beta_{0}+\varepsilon_{1}\right) a^{2}(1) \zeta_{1}}{\left(a^{2}(1)+\zeta^{2}\right)^{3 / 2}}, \phi_{1}, \frac{z_{1}}{1+a(s) \phi_{s}}-\frac{a(s) z \phi_{1 s}}{\left(1+a(s) \phi_{s}\right)^{2}}\right) .
\end{aligned}
$$

An explicit calculation shows that the above change of variable transforms equations (27)(30) into

$$
\begin{aligned}
\rho_{x} & =-a^{-1}(1) \zeta, \\
\zeta_{x} & =-\frac{\left(a^{2}(1)+\zeta^{2}\right)^{3 / 2}}{a^{2}(1)\left(\beta_{0}+\varepsilon_{1}\right)}\left(\frac{z^{2}(1)+a^{2}(1)}{2\left(1+a(1) \phi_{s}(1)\right)^{2}}+\left(\alpha_{0}+\varepsilon_{2}\right) \rho-\frac{\mu^{\star}}{2}\right), \\
\phi_{x} & =-a^{-1}(s) z, \\
z_{x} & =-\frac{a(s) z\left(a^{-1}(s) z\right)_{s}}{1+a(s) \phi_{s}}-\left(1+a(s) \phi_{s}\right)\left(\left(\frac{z^{2}+a^{2}(s)}{2\left(1+a(s) \phi_{s}\right)^{2}}\right)_{s}-\gamma(-s)\right) ;
\end{aligned}
$$


these equations hold in the phase space $X$ and the domain of the vector field on their right-hand side is a neighbourhood $N$ of the origin in the linear space

$$
Z=\{(\rho, \zeta, \phi, z) \in Y: z(0)=0, z(1)=\zeta\}
$$

Notice that the change of variable preserves the revsersibility; the action of the reverser $S$ : $X \rightarrow X$ is given by $S(\rho, \zeta, \phi, z)=(\rho,-\zeta, \phi,-z)$. Equations (36)-(39) represent Hamilton's equations for the Hamiltonian system $\left(X, \Phi^{\varepsilon}, K^{\varepsilon}\right)$, where

$$
\begin{aligned}
& \left.\Phi^{\varepsilon}\right|_{(\rho, \zeta, \phi, z)}\left(\left(\rho_{1}, \zeta_{1}, \phi_{1}, z_{1}\right),\left(\rho_{2}, \zeta_{2}, \phi_{2}, z_{2}\right)\right)= \\
& \quad-\frac{\left(\beta_{0}+\varepsilon_{1}\right) a^{2}(1)}{\left(\zeta^{2}+a^{2}(1)\right)^{3 / 2}}\left(\zeta_{2} \rho_{1}-\zeta_{1} \rho_{2}\right)+\int_{0}^{1}\left\{\frac{z_{2} \phi_{1}-z_{1} \phi_{2}}{1+a(s) \phi_{s}}-\frac{a(s) z}{\left(1+a(s) \phi_{s}\right)^{2}}\left(\phi_{2 s} \phi_{1}-\phi_{1 s} \phi_{2}\right)\right\} \mathrm{d} s
\end{aligned}
$$

and $K^{\varepsilon} \in C^{\infty}(V, \mathbb{R})$ is defined by the formula

$$
\begin{aligned}
K^{\varepsilon}(\rho, \zeta, \phi, z)=\int_{0}^{1}\left\{\frac{a^{2}(s)-z^{2}}{2\left(1+a(s) \phi_{s}\right)}+\Gamma(s) \phi_{s}-\frac{a(s)}{2}\right\} \mathrm{d} s \\
-\frac{1}{2}\left(\alpha_{0}+\varepsilon_{2}\right) \rho^{2}+\frac{1}{2} \mu^{\star} \rho-\frac{a(1)\left(\beta_{0}+\varepsilon_{1}\right)}{\sqrt{a^{2}(1)+\zeta^{2}}}+\left(\beta_{0}+\varepsilon_{1}\right) .
\end{aligned}
$$

The next step is to verify that equations (36)-(39) satisfy the hypotheses of Theorem 3.1. We write these equations as

$$
u_{x}=L u+N^{\varepsilon}(u)
$$

in which the linear operator $L: \mathcal{D}(L) \subset X \rightarrow X$ with $\mathcal{D}(L)=Z$ is given by

$$
L\left(\begin{array}{c}
\rho \\
\zeta \\
\phi \\
z
\end{array}\right)=\left(\begin{array}{c}
-a^{-1}(1) \zeta \\
a^{4}(1) \beta_{0}^{-1} \phi_{s}(1)-a(1) \alpha_{0} \beta_{0}^{-1} \rho \\
-a^{-1}(s) z \\
\left(a^{3}(s) \phi_{s}\right)_{s}
\end{array}\right)
$$

(the linearisation of the Hamiltonian vector field $v_{K^{\varepsilon}}$ at $\varepsilon=0$ ). It follows from Proposition 3.3 and Lemma 3.4, the former of which is proved using the elementary theory of ordinary differential equations, that $L$ satisfies hypotheses (H1) and (H2); hypothesis (H3) is clearly satisfied for an arbitrary value of $k$.

Proposition 3.3 The spectrum of the operator $L: \mathcal{D}(L) \subset X \rightarrow X$ consists of isolated, geometrically simple eigenvalues of finite algebraic multiplicity.

Lemma 3.4 There exist real constants $C, \xi_{0}>0$ such that each solution $v \in Y$ of the resolvent equation

$$
(L-\mathrm{i} \xi I) v=f^{\star},
$$

in which $f^{\star}$ belongs to $X$ and $\xi$ is a real number with $|\xi|>\xi_{0}$, satisfies the estimates

$$
\|v\|_{Y} \leq C\left\|f^{\star}\right\|_{X}, \quad\|v\|_{X} \leq \frac{C}{|\xi|}\left\|f^{\star}\right\|_{X} .
$$


Proof. Let us write the resolvent equation (42) in the form

$$
\begin{aligned}
-a^{-1}(1) \zeta-\mathrm{i} \xi \rho & =\rho^{\star}, \\
a^{4}(1) \beta_{0}^{-1} \phi_{s}(1)-a(1) \alpha_{0} \beta_{0}^{-1} \rho-\mathrm{i} \xi \zeta & =\zeta^{\star}, \\
-a^{-1}(s) z-\mathrm{i} \xi \phi & =\phi^{\star}, \\
\left(a^{3}(s) \phi_{s}\right)_{s}-\mathrm{i} \xi z & =z^{\star},
\end{aligned}
$$

where $\left(\rho^{\star}, \omega^{\star}, \phi^{\star}, z^{\star}\right) \in X$ and $(\rho, \omega, \phi, z) \in Z$, so that $\phi^{\star}(0)=0, \phi^{\star}(1)=\rho^{\star}$ and $\phi(0)=0$, $\phi(1)=\rho, z(0)=0, z(1)=\zeta$.

The first step is to differentiate (45) and multiply by $a^{3 / 2}(s)$, and multiply (46) by $a^{-1 / 2}(s)$, so that

$$
\begin{aligned}
-a^{3 / 2}(s)\left(a^{-1}(s) z\right)_{s}-\mathrm{i} \xi a^{3 / 2}(s) \phi_{s} & =a^{3 / 2}(s) \phi_{s}^{\star}, \\
a^{-1 / 2}(s)\left(a^{3}(s) \phi_{s}\right)_{s}-\mathrm{i} \xi a^{-1 / 2}(s) z & =a^{-1 / 2}(s) z^{\star}
\end{aligned}
$$

squaring and adding these equations, one finds that

$$
\begin{aligned}
& a^{3}(s)\left|\left(a^{-1}(s) z\right)_{s}\right|^{2}+a^{-1}(s)\left|\left(a^{3}(s) \phi_{s}\right)_{s}\right|+\xi^{2}\left(a^{3}(s)\left|\phi_{s}\right|^{2}+a^{-1}(s)|z|^{2}\right) \\
& \quad+2 \xi \operatorname{Im}\left\{\left(a^{-1}(s) z\right)_{s} a^{3}(s) \bar{\phi}_{s}+a^{-1}(s) z\left(a^{3}(s) \bar{\phi}_{s}\right)_{s}\right\}=a^{3}(s)\left|\phi_{s}^{\star}\right|^{2}+a^{-1}(s)\left|z^{\star}\right|^{2} .
\end{aligned}
$$

Let $\|\cdot\|$ and $\|\cdot\|_{w}$ denote respectively the usual $L^{2}(0,1)$-norm and the $L^{2}(0,1)$-norm with weight function $w$. We integrate the above equation over $(0,1)$, so that

$$
\begin{array}{r}
\left\|\left(a^{-1}(s) z\right)_{s}\right\|_{a^{3}}^{2}+\left\|\left(a^{3}(s) \phi_{s}\right)_{s}\right\|_{a^{-1}}^{2}+\xi^{2}\left(\left\|\phi_{s}\right\|_{a^{3}}^{2}+\|z\|_{a^{-1}}^{2}\right) \\
=\left\|\phi_{s}^{\star}\right\|_{a^{3}}^{2}+\left\|z^{\star}\right\|_{a^{-1}}^{2}-2 \xi a^{2}(1) \operatorname{Im}\left\{z(1) \bar{\phi}_{s}(1)\right\},
\end{array}
$$

and multiplying (44) by $\bar{\phi}_{s}(1)$ and taking real parts, we find that

$$
\begin{aligned}
\xi \operatorname{Im}\left\{z(1) \bar{\phi}_{s}(1)\right\} & =-\frac{a^{4}(1)}{\beta_{0}}\left|\phi_{s}(1)\right|^{2}+\frac{a(1) \alpha_{0}}{\beta_{0}} \operatorname{Re}\left\{\phi(1) \bar{\phi}_{s}(1)\right\}+\operatorname{Re}\left\{\zeta^{\star} \bar{\phi}_{s}(1)\right\} \\
& \leq C_{1}\left(\left|\phi_{s}(1)\right|^{2}+|\phi(1)|^{2}+\left|\zeta^{\star}\right|^{2}\right) .
\end{aligned}
$$

To estimate $\left|\phi_{s}(1)\right|^{2}$ we multiply (46) by $s^{n}$, where $n$ is a positive integer, and integrate over $(0,1)$, so that

$$
a^{3}(1) \phi_{s}(1)=\int_{0}^{1} a^{3}(s) \phi_{s}(s) n s^{n-1} \mathrm{~d} s+\mathrm{i} \xi \int_{0}^{1} z(s) s^{n} \mathrm{~d} s+\int_{0}^{1} z^{\star}(s) s^{n} \mathrm{~d} s,
$$

and an application of the Cauchy-Schwarz inequality yields

$$
\left|\phi_{s}(1)\right|^{2} \leq C_{2}\left(\frac{n^{2}}{2 n-1}\left\|\phi_{s}\right\|_{a^{6}}^{2}+\frac{\xi^{2}}{2 n+1}\|z\|^{2}+\frac{1}{2 n+1}\left\|z^{\star}\right\|^{2}\right) .
$$

A straightforward combination of estimates (47)-(49), the inequality $|\phi(1)|^{2} \leq\left\|\phi_{s}\right\|^{2}$ and the fact that $\|\cdot\|$ and $\|\cdot\|_{w}$ are equivalent norms for any positive $w \in C[0,1]$ shows that

$$
\begin{aligned}
& \left\|\phi_{s s}\right\|^{2}+\left\|\phi_{s}\right\|^{2}+\left\|z_{s}\right\|^{2}+\|z\|^{2}+\xi^{2}\left(\left\|\phi_{s}\right\|^{2}+\|z\|^{2}\right) \\
& \quad \leq C_{3}\left(\frac{n^{2}}{2 n-1}\left\|\phi_{s}\right\|^{2}+\frac{\xi^{2}}{2 n+1}\|z\|^{2}+\left\|\phi_{s}^{\star}\right\|^{2}+\left\|z^{\star}\right\|^{2}+\left|\zeta^{\star}\right|^{2}\right) .
\end{aligned}
$$


Choosing $n$ large enough so that $C_{3} /(2 n+1) \leq 1 / 2$, we thefore find that

$$
\left\|\phi_{s s}\right\|^{2}+\left\|\phi_{s}\right\|^{2}+\left\|z_{s}\right\|^{2}+\|z\|^{2}+\frac{\xi^{2}}{2}\left(\left\|\phi_{s}\right\|^{2}+\|z\|^{2}\right) \leq C_{3}\left(\left|\zeta^{\star}\right|^{2}+\left\|\phi_{s}^{\star}\right\|^{2}+\left\|z^{\star}\right\|^{2}\right)
$$

and hence that

$$
\|\phi\|_{H^{2}}^{2}+\|z\|_{H^{1}}^{2}+\frac{\xi^{2}}{2}\left(\|\phi\|_{H^{1}}^{2}+\|z\|^{2}\right) \leq C_{4}\left(\left|\zeta^{\star}\right|^{2}+\left\|\phi^{\star}\right\|_{H^{1}}^{2}+\left\|z^{\star}\right\|^{2}\right)
$$

for sufficiently large $\xi$, where we have exploited the fact that $\phi(0), \phi^{\star}(0)$ and $z(0)$ all vanish.

It follows from (44) that

$$
\xi^{2}|\zeta|^{2} \leq C_{4}\left(\left|\zeta^{\star}\right|^{2}+|\phi(1)|^{2}+\left|\phi_{s}(1)\right|^{2}\right) \leq C_{5}\left(\left|\zeta^{\star}\right|^{2}+\|\phi\|_{H^{1}}^{2}+\xi^{2}\|z\|^{2}+\left\|z^{\star}\right\|^{2}\right)
$$

and clearly $|\rho|^{2}=|\phi(1)|^{2} \leq\|\phi\|_{H^{1}}^{2}$, so that altogether

$$
\|\phi\|_{H^{2}}^{2}+\|z\|_{H^{1}}^{2}+\frac{\xi^{2}}{2}\left(|\rho|^{2}+|\zeta|^{2}+\|\phi\|_{H^{1}}^{2}+\|z\|^{2}\right) \leq C_{6}\left(\left|\zeta^{\star}\right|^{2}+\left\|\phi^{\star}\right\|_{H^{1}}^{2}+\left\|z^{\star}\right\|^{2}\right) .
$$

\subsection{Eigenvalues of the linearised problem}

In this section we examine the spectrum of $L$ in more detail, in particular the qualitative dependence of its eigenvalues upon $\left(\beta_{0}, \alpha_{0}\right)$. Eliminating $\rho, \zeta$ and $z$, we find that the eigenvalue problem $L u=\kappa u$ is equivalent to

$$
\begin{aligned}
-a^{-1}(s)\left(a^{3}(s) \phi_{s}\right)_{s} & =\kappa^{2} \phi, \quad 0<s<1, \\
-\frac{a^{3}(1)}{\beta} \phi_{s}(1)+\frac{\alpha}{\beta} \phi(1) & =\kappa^{2} \phi(1), \\
\phi(0) & =0
\end{aligned}
$$

(for convenience we drop the subscript 0 in this section). The change of variable

$$
y=\int_{0}^{s} a^{-1}(u) \mathrm{d} u, \quad v(y)=a(s) \phi(s)
$$

transforms the above equations into the equivalent non-selfadjoint Sturm-Liouville problem

$$
\begin{aligned}
-v_{y y}+Q(y) v & =\nu v, \\
\frac{v_{y}(1)}{v(1)} & =\hat{\alpha}-\hat{\beta} \nu, \\
v(0) & =0,
\end{aligned}
$$

where $\nu=\kappa^{2}, Q(y)=-\gamma^{\prime}(-s), \hat{\alpha}=a^{\prime}(1)+a^{-2}(1) \alpha$ and $\hat{\beta}=a^{-2}(1) \beta$; detailed spectral results for problems of this type have been presented by Binding, Browne, Code \& Watson [4].

The Sturm-Liouville problem (53)-(55) has a countable number of geometrically simple eigenvalues which can be listed as $\nu_{0}, \nu_{1}, \nu_{2}, \ldots$, where $\operatorname{Re} \nu_{n} \leq \operatorname{Re} \nu_{n+1}$ (entries are repeated according to their algebraic multiplicity); they occur in complex-conjugate pairs and satisfy

$$
\nu_{n}=n^{2} \pi^{2}+\int_{0}^{1} Q(y) \mathrm{d} y-\frac{2 \hat{\alpha}}{\hat{\beta}}+o\left(\frac{1}{n}\right), \quad n \in \mathbb{N}_{0} .
$$


Observe that the real eigenvalues of the spectral problem (53)-(55) correspond to the intersections in the $(\nu, s)$ plane of the line $s=\hat{\alpha}-\hat{\beta} \nu$ and the curve $s=B(\nu)$, where $B(\nu)=$ $v_{y}(1 ; \nu) / v(1 ; \nu)$ and $v(y ; \nu)$ solves the initial-value problem

$$
-v_{y y}+Q(y) v=\nu v, \quad v(0 ; \nu)=0
$$

a tangent intersection indicates that the eigenvalue has algebraic multiplicity 2 . The function $B(\nu)=v_{y}(1 ; \nu) / v(1 ; \nu)$ has poles exactly at the Dirichlet eigenvalues $\nu_{n}^{\mathrm{D}}$ (the necessarily real, positive and algebraically simple eigenvalues of the self-adjoint problem in which (54) is replaced by $v(1)=0)$; it is strictly decreasing from $+\infty$ to $-\infty$ in each interval $\left(-\infty, \nu_{0}^{\mathrm{D}}\right)$ and $\left(\nu_{k}^{\mathrm{D}}, \nu_{k+1}^{\mathrm{D}}\right), k \in \mathbb{N}$. It follows that (53)-(55) has at least one real eigenvalue in each interval $\left(\nu_{k}^{\mathrm{D}}, \nu_{k+1}^{\mathrm{D}}\right), k \in \mathbb{N}$ (see Figure 2).

Further information concerning the distribution of the eigenvalues $\nu_{n}$ is obtained by comparing (56) with the corresponding formula

$$
\nu_{n}^{\mathrm{D}}=(n+1)^{2} \pi^{2}+\int_{0}^{1} Q(y) \mathrm{d} y+o\left(\frac{1}{n}\right), \quad n \in \mathbb{N}_{0}
$$

for the Dirichlet eigenvalues; in particular one finds that

$$
\nu_{n-1}^{\mathrm{D}}<\operatorname{Re} \nu_{n+1}<\nu_{n}^{\mathrm{D}}
$$

for sufficiently large $n$. It follows that for sufficiently large $n$ the eigenvalue $\nu_{n+1}$ is real and located in the interval $\left(\nu_{n-1}^{\mathrm{D}}, \nu_{n}^{\mathrm{D}}\right)$. Using this observation and the above geometrical characterisation of real eigenvalues, one concludes that

(i) each interval $\left(\nu_{n-1}^{\mathrm{D}}, \nu_{n}^{\mathrm{D}}\right), n \in \mathbb{N}$ contains a simple real eigenvalue;

(ii) there are precisely two additional eigenvalues (counted according to algebraic multiplicity) which are either

(a) real, and located to the left of $\nu_{0}^{\mathrm{D}}$ (Figure 2 (top left)),

(b) real, and located in the interval $\left(\nu_{n-1}^{\mathrm{D}}, \nu_{n}^{\mathrm{D}}\right)$ for some $n \in \mathbb{N}$ (Figure 2 (top right)),

(c) a complex-conjugate pair (with non-vanishing imaginary part) whose real part is smaller than $\nu_{0}^{\mathrm{D}}$ (Figure 2 (bottom)),

(d) a complex-conjugate pair (with non-vanishing imaginary part) whose real part lies in the interval $\left(\nu_{n-1}^{\mathrm{D}}, \nu_{n}^{\mathrm{D}}\right)$ for some $n \in \mathbb{N}$ (Figure 2 (bottom)).

The eigenvalues $\kappa$ of $L$ are recovered from the above analysis by the formula $\nu=\kappa^{2}$, so that in particular they occur in plus-minus pairs. Clearly $L$ has a real eigenvalue in each interval $\left(\left(\nu_{n-1}^{\mathrm{D}}\right)^{1 / 2},\left(\nu_{n}^{\mathrm{D}}\right)^{1 / 2}\right)$ and $\left(-\left(\nu_{n}^{\mathrm{D}}\right)^{1 / 2},-\left(\nu_{n-1}^{\mathrm{D}}\right)^{1 / 2}\right), n \in \mathbb{N}$ (see point (i) above), and it follows from point (ii) that there are four additional eigenvalues (counted according to algebraic multiplicity). The four additional eigenvalues could be real with magnitude greater than $\left(\mu_{0}^{\mathrm{D}}\right)^{1 / 2}$ (case (b)) or a plus-minus, complex-conjugate quartet with nonvanishing real and imaginary parts (cases (c) and (d)). Observe that only the remaining case (a) can lead to purely imaginary eigenvalues of $L$, and we now examine this case in detail. 

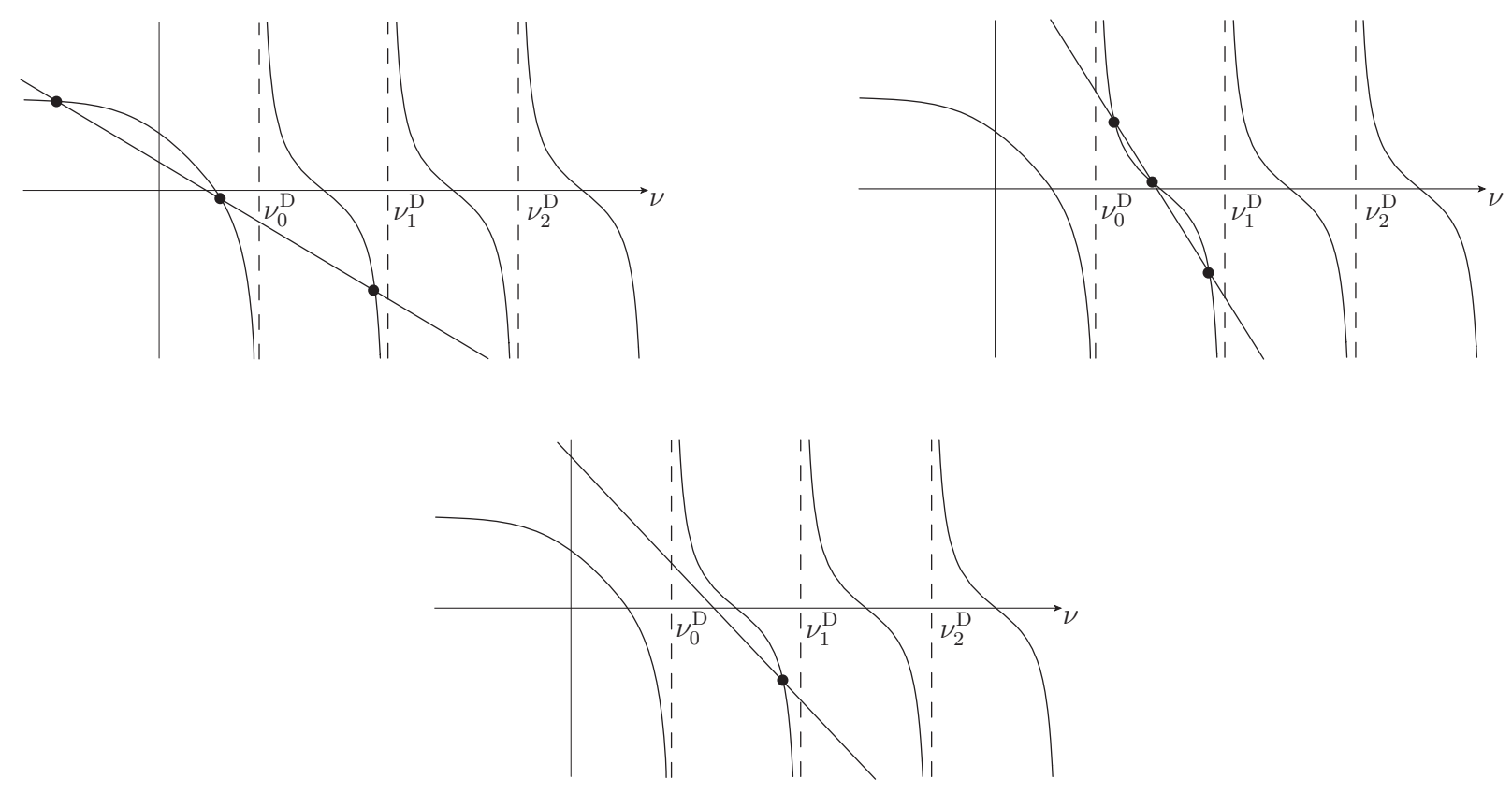

Figure 2: Geometric characterisation of the eigenvalues $\nu_{n}$ as the points of intersection of the curve $s=B(\nu)$ with the straight line $\nu=\hat{\alpha}-\hat{\beta} \nu$; one real eigenvalue lies in each interval $\left(\nu_{n-1}^{\mathrm{D}}, \nu_{n}^{\mathrm{D}}\right), n \in \mathbb{N}$. Clockwise from top left: two additional real eigenvalues to the left of $\nu_{0}^{\mathrm{D}}$; two additional real eigenvalues in the interval $\left(\nu_{n-1}^{\mathrm{D}}, \nu_{n}^{\mathrm{D}}\right)$ for some $n \in \mathbb{N}$; two additional complex eigenvalues.

Case (a) has eight subcases, each of which is illustrated in Figure 3. Clearly a positive eigenvalue $\nu$ yields a plus-minus pair of real eigenvalues of $L$, while a negative eigenvalue $\nu$ yields a complex-conjugate pair of purely imaginary eigenvalues of $L$; an algebraically double positive or negative eigenvalue $\nu$ yields a pair of algebraically double real or purely imaginary eigenvalues. A zero eigenvalue of (53)-(55) similarly leads to a zero eigenvalue of $L$, the algebraic multiplicity of which is readily determined by studying the equation $L u=0$ directly. A straightforward calculation shows that zero is an eigenvalue of $L$ if and only if $\alpha=\alpha_{\star}$; the eigenvalue has algebraic multiplicity 2 when $\beta \neq \beta_{\star}$ and algebraic multiplicity 4 when $\beta=\beta_{\star}$, where

$$
\alpha_{\star}=\left(\int_{0}^{1} a^{-3}(s) \mathrm{d} s\right)^{-1}, \quad \beta_{\star}=\alpha_{\star}^{2} \int_{0}^{1} a(s)\left(\int_{0}^{s} a^{-3}(t) \mathrm{d} t\right)^{2} \mathrm{~d} s .
$$

The generalised eigenvectors $w_{j}$, where $L w_{1}=0, L w_{2}=w_{1}$ and $L w_{j}=w_{j-1}, j=3,4$ for $\beta=\beta_{\star}$ are given by

$$
w_{1}=\left(\begin{array}{c}
\int_{0}^{1} a^{-3}(s) \mathrm{d} s \\
0 \\
\int_{0}^{s} a^{-3}(t) \mathrm{d} t \\
0
\end{array}\right), \quad w_{2}=\left(\begin{array}{c}
0 \\
-a(1) \int_{0}^{1} a^{-3}(s) \mathrm{d} s \\
0 \\
-a(s) \int_{0}^{s} a^{-3}(t) \mathrm{d} t
\end{array}\right),
$$



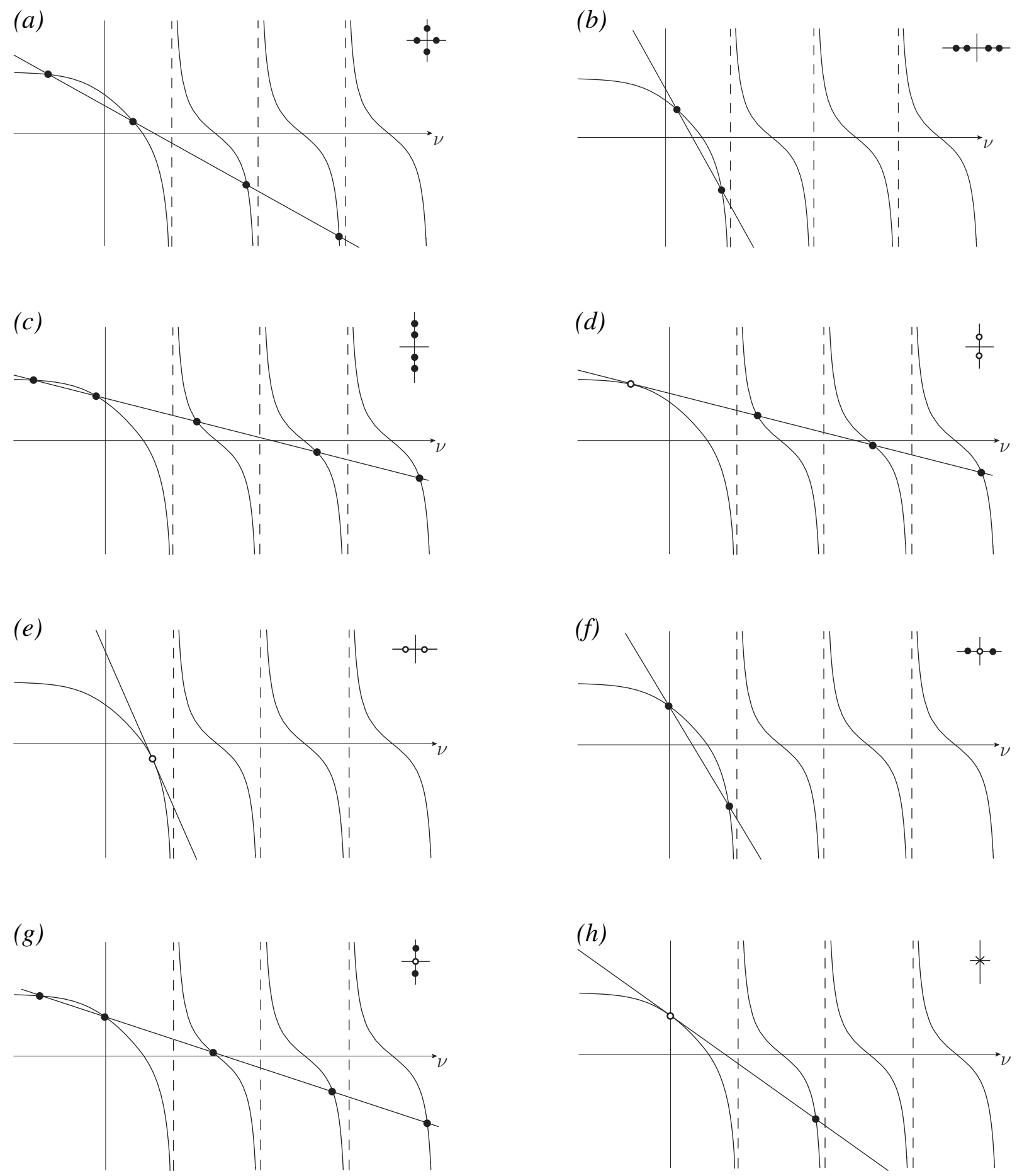

Figure 3: The eight cases in which the Sturm-Liouville problem (53)-(55) has two real eigenvalues (counted according to algebraic multiplicity) which are less than $\nu_{0}^{\mathrm{D}}$; solid and hollow dots denote respectively algebraically simple and algebraically double eigenvalues. The insets show the corresponding eigenvalues of the linear operator $L$; the cross denotes an eigenvalue of algebraic multiplicity 4. 


$$
\begin{gathered}
w_{3}=\left(\begin{array}{c}
-\int_{0}^{1} a^{-3}(s) \int_{0}^{s} a(t) \int_{0}^{t} a^{-3}(u) \mathrm{d} u \mathrm{~d} t \mathrm{~d} s \\
0 \\
-\int_{0}^{s} a^{-3}(t) \int_{0}^{t} a(u) \int_{0}^{u} a^{-3}(v) \mathrm{d} v \mathrm{~d} u \mathrm{~d} t \\
0
\end{array}\right), \\
w_{4}=\left(\begin{array}{c}
a \\
a(1) \int_{0}^{1} a^{-3}(s) \int_{0}^{s} a(t) \int_{0}^{t} a^{-3}(u) \mathrm{d} u \mathrm{~d} t \mathrm{~d} s \\
0 \\
a(s) \int_{0}^{s} a^{-3}(t) \int_{0}^{t} a(u) \int_{0}^{u} a^{-3}(v) \mathrm{d} v \mathrm{~d} u \mathrm{~d} t
\end{array}\right) .
\end{gathered}
$$

Recall that $L$ has four eigenvalues (counted according to algebraic multiplicity) with real part in the interval $\left(-\left(\nu_{0}^{\mathrm{D}}\right)^{1 / 2},\left(\nu_{0}^{\mathrm{D}}\right)^{1 / 2}\right)$. An algebraically simple zero eigenvalue of (53)-(55) (Figure $3(\mathrm{f})$ or $(\mathrm{g}))$ therefore corresponds to the case $\alpha=\alpha_{\star}, \beta \neq \beta_{\star}$, while an algebraically double zero eigenvalue (Figure 3(h)) corresponds to the case $\alpha=\alpha_{\star}, \beta=\beta_{\star}$; it follows that

$$
B(0)=a^{\prime}(1)+a^{-2}(1) \alpha_{\star}, \quad B^{\prime}(0)=-a^{-2}(1) \beta_{\star} .
$$

From this observation we conclude that Figure 3(f) corresponds to the case $\alpha=\alpha_{\star}, \beta>\beta_{\star}$ while Figure 3(g) corresponds to the case $\alpha=\alpha_{\star}, \beta<\beta_{\star}$; futhermore Figure 3(a) corresponds to the case $\alpha<\alpha_{\star}$, Figures 3(b) and (e) arise when $\alpha>\alpha_{\star}, \beta>\beta_{\star}$ and Figures 3(c) and (d) arise when $\alpha>\alpha_{\star}, \beta<\beta_{\star}$.

Figure 4 summarises the behaviour of the four eigenvalues of $L$ whose real parts lie in $\left(-\left(\nu_{0}^{\mathrm{D}}\right)^{1 / 2},\left(\nu_{0}^{\mathrm{D}}\right)^{1 / 2}\right)$. The values of $\beta$ and $\alpha$ determine respectively the gradient of the straight line in Figure 3 and its point of intersection with the vertical axis: increasing $\beta$ makes it steeper, while increasing $\alpha$ moves it upwards. Suppose for example that $\alpha<\alpha_{\star}$ (Figure 3(a)), and we increase the value of $\alpha$ while keeping $\beta$ fixed; according to whether $\beta<\beta_{\star}$ or $\beta>\beta_{\star}$ we pass through the sequence (a), (g), (c), (d) or (a), (f), (b), (e). Figure 4 follows from these observations. Of particular interest are the four bifurcation curves $C_{j}, j=1,4$, at points of which the qualitative nature of the eigenvalue picture changes. In contrast to $C_{4}=\left\{\left(\beta, \alpha_{\star}\right): \beta>\beta_{\star}\right\}$ and $C_{3}=\left\{\left(\beta, \alpha_{\star}\right): \beta<\beta_{\star}\right\}$ explicit parameterisations of $C_{2}$ and $C_{1}$ are available only in certain special cases, in particular for irrotational flows (Kirchgässner [29]).

Recall that $L$ also has a countably infinite number of eigenvalues whose real parts lie outside the range $\left(-\left(\nu_{0}^{\mathrm{D}}\right)^{1 / 2},\left(\nu_{0}^{\mathrm{D}}\right)^{1 / 2}\right)$; these eigenvalues are all real and simple at points in the $(\beta, \alpha)$ parameter plane below the curve $C_{1} \cup C_{2}$, while all but possibly four of the complete set of eigenvalues are real above $C_{1} \cup C_{2}$. The region above $C_{1} \cup C_{2}$ contains a further countably infinite family of bifurcation curves, points of the $n$th of which correspond to the transition between Figure 2(top right) and Figure 2(bottom): four real eigenvalues with magnitudes in the interval $\left(\left(\nu_{n-1}^{\mathrm{D}}\right)^{1 / 2},\left(\nu_{n}^{\mathrm{D}}\right)^{1 / 2}\right)$ become complex by colliding in pairs on the real axis. At each point in $(\beta, \alpha)$ parameter space above $C_{1} \cup C_{2}$ there is therefore an infinite number of real, simple eigenvalues and possibly one pair of algebraically double real eigenvalues or one plus-minus, complex-conjugate quartet of complex eigenvalues. 


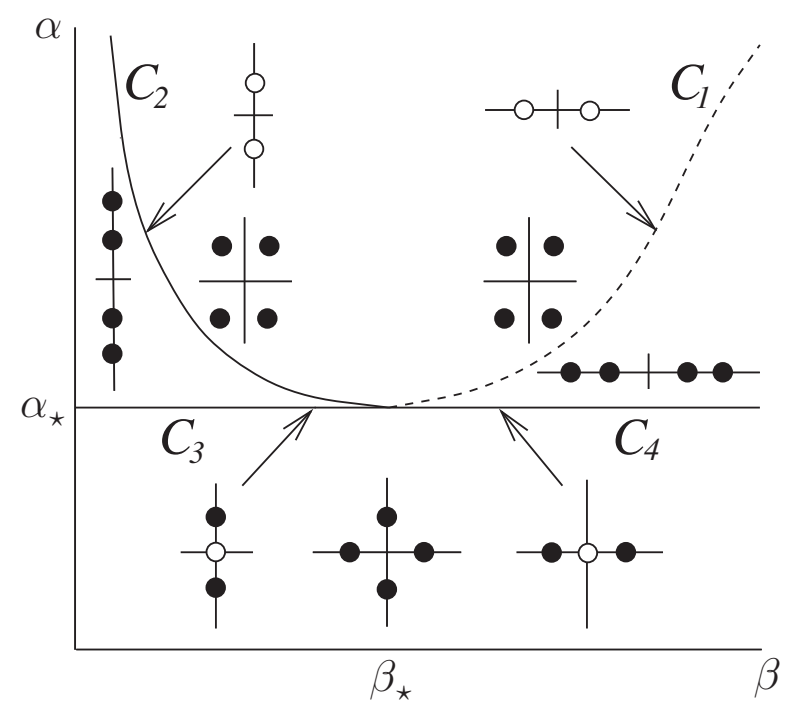

Figure 4: Eigenvalues of $L$ whose real parts lie in $\left(-\left(\nu_{0}^{\mathrm{D}}\right)^{1 / 2},\left(\nu_{0}^{\mathrm{D}}\right)^{1 / 2}\right)$; solid and hollow dots denote respectively algebraically simple and double eigenvalues. The curves $C_{j}, j=1, \ldots, 4$ consist of points in $(\beta, \alpha)$ parameter space at which the qualitative nature of the eigenvalue picture changes; the real parts of the four complex eigenvalues just above $C_{1} \cup C_{2}$ move out of the range $\left(-\left(\nu_{0}^{\mathrm{D}}\right)^{1 / 2},\left(\nu_{0}^{\mathrm{D}}\right)^{1 / 2}\right)$ as one moves away from $C_{1} \cup C_{2}$.

The spectral theory presented above can also be formulated in terms of self-adjoint operators on Pontryagin spaces (see e.g. Bognar [5] and Iohvidov, Krein \& Langer [22]). Introduce the $\pi_{1}$-space $P=L^{2}(0,1) \times \mathbb{R}$ with indefinite inner product

$$
\left[\left(\phi_{1}, b_{1}\right),\left(\phi_{2}, b_{2}\right)\right]=\left\langle a \phi_{1}, \phi_{2}\right\rangle_{L^{2}(0,1)}-\beta b_{1} b_{2}
$$

and the linear operator $T: \mathcal{D}(T) \subset P \rightarrow P$ defined by

$$
T(\phi, b)=\left(-a^{-1}(s)\left(a^{3}(s) \phi_{s}\right)_{s},-a^{3}(1) \beta^{-1} \phi_{s}(1)+\alpha \beta^{-1} \phi(1)\right)
$$

with

$$
\mathcal{D}(T)=\left\{(\phi, b): \phi \in H^{2}(0,1), \phi(0)=0, \phi(1)=b\right\} .
$$

Observe that $T$ is densely defined (with respect to the topology of $L^{2}(0,1) \times \mathbb{R}$ ) and symmetric (with respect to $[\cdot, \cdot]$ ), so that it is a self-adjoint operator on $P$, and it follows from (50)-(52) that $\kappa$ is an eigenvalue of $L$ if and only if $\kappa^{2}$ is an eigenvalue of $T$. This framework, which was used by Wahlén [35] in his study of periodic water waves, yields a convenient method of calculating the local parts of the curves $C_{1}$ and $C_{2}$ in Figure 4 near the point $\left(\beta_{\star}, \alpha_{\star}\right)$.

A point $\left(\beta_{\kappa}, \alpha_{\kappa}\right)$ of $C_{1}$ is characterised by the fact that $\kappa^{2}$ is a geometrically simple eigenvalue of $T_{\kappa}:=\left.T\right|_{(\beta, \alpha)=\left(\beta_{\kappa}, \alpha_{\kappa}\right)}$ with algebraic multiplicity 2 , and since the algebraic multiplicity of this eigenvalue exceeds its geometric multiplicity the corresponding eigenspace $E_{\kappa^{2}}$ is neutral, that is $[\tilde{v}, \tilde{v}]_{\kappa}:=[\tilde{v}, \tilde{v}]_{\beta=\beta_{\kappa}}$ vanishes for every $\tilde{v}=(v, v(1)) \in E_{\kappa^{2}}$ (see Wahlén [35, p. 12]). One obtains expressions for $v_{j}, \beta_{j}, \alpha_{j}$ by inserting the expansions

$$
T_{\kappa}=T_{0}+\kappa^{2} T_{2}+\kappa^{4} T_{4}+\ldots, \quad[\cdot, \cdot]_{\kappa}=[\cdot, \cdot]_{0}+\kappa^{2}[\cdot, \cdot]_{2}+\kappa^{4}[\cdot, \cdot]_{4}+\ldots
$$


and

$$
\begin{gathered}
v_{\kappa}=v_{0}+\kappa^{2} v_{2}+\kappa^{4} v_{4}+\ldots, \\
\beta_{\kappa}=\beta_{\star}+\kappa^{2} \beta_{2}+\kappa^{4} \beta_{4}+\ldots, \quad \alpha_{\kappa}=\alpha_{\star}+\kappa^{2} \alpha_{2}+\kappa^{4} \alpha_{4}+\ldots
\end{gathered}
$$

into

$$
T_{\kappa} \tilde{v}_{\kappa}=\kappa^{2} \tilde{v}_{\kappa}, \quad\left[\tilde{v}_{\kappa}, \tilde{v}_{\kappa}\right]_{\kappa}=0
$$

and equating coefficients of $\kappa^{2}$. In particular we find that

$$
\begin{gathered}
v_{0}=\int_{0}^{s} a^{-3}(s) \mathrm{d} t, \quad v_{2}=-\int_{0}^{s} a^{-3}(t) \int_{0}^{t} a(u) \int_{0}^{u} a^{-3}(v) \mathrm{d} v \mathrm{~d} u \mathrm{~d} t, \\
\beta_{2}=\frac{2\left[\tilde{v}_{2}, \tilde{v}_{0}\right]_{0}}{v_{0}^{2}(1)}, \quad \alpha_{2}=0, \quad \alpha_{4}=\frac{\left[\tilde{v}_{2}, \tilde{v}_{0}\right]_{0}}{v_{0}^{2}(1)}
\end{gathered}
$$

and hence that

$$
\alpha_{4}=\alpha_{\star}^{2} d_{1}, \quad \beta_{2}=2 \alpha_{\star}^{2} d_{1},
$$

where

$$
\begin{aligned}
d_{1}=-\alpha_{\star}\left(\int_{0}^{1}\right. & \left.a^{-3}(s) \int_{0}^{s} a(t) \int_{0}^{t} a^{-3}(u) \mathrm{d} u \mathrm{~d} t \mathrm{~d} s\right)^{2} \\
& +\int_{0}^{1} a^{-3}(s)\left(\int_{0}^{s} a(t) \int_{0}^{t} a^{-3}(u) \mathrm{d} u \mathrm{~d} t\right)^{2} \mathrm{~d} s>0 .
\end{aligned}
$$

A similar calculation shows that the corresponding parameterisation of the local part of $C_{2}$ near $\left(\beta_{\star}, \alpha_{\star}\right)$ is

$$
\beta_{\lambda}=\beta_{\star}-2 \alpha_{\star}^{2} d_{1} \lambda^{2}+O\left(\lambda^{4}\right), \quad \alpha_{\lambda}=\alpha_{\star}+\alpha_{\star}^{2} d_{1} \lambda^{4}+O\left(\lambda^{6}\right)
$$

(a point $\left(\beta_{\lambda}, \alpha_{\lambda}\right)$ of $C_{2}$ is characterised by the fact that $-\lambda^{2}$ is a geometrically simple eigenvalue of $T_{\lambda}:=\left.T\right|_{(\beta, \alpha)=\left(\beta_{\lambda}, \alpha_{\lambda}\right)}$ with algebraic multiplicity 2$)$.

\section{The reduced Hamiltonian systems}

Our existence theory for solitary waves is completed by showing that the reduced Hamiltonian system on the centre manifold admits homoclinic solutions. Irrotational solitary waves have been found at points in $(\beta, \alpha)$ parameter space near $C_{1}, C_{2}$ and $C_{4}$ in this fashion, and in this section we examine the corresponding reduced systems in our more general context.

\subsection{Homoclinic bifurcation at $C_{4}$}

A Hamiltonian $0^{2}$ resonance takes place at points of the curve $C_{4}$ in Figure 4: two real eigenvalues become purely imaginary by colliding at the origin and forming a Jordan chain of length

2. This resonance is associated with the bifurcation of a branch of homoclinic solutions into the region with real eigenvalues (the parameter regime marked I in Figure 1(a)). Let us therefore fix reference values $\left(\beta_{0}, \alpha_{0}\right) \in C_{4}$, so that $\beta_{0}>\beta_{\star}, \alpha_{0}=\alpha_{\star}$, and introduce a bifurcation parameter by choosing $\left(\varepsilon_{1}, \varepsilon_{2}\right)=(0, \delta)$, where $0<\delta \ll 1$. 
Formulae for the generalised eigenvectors $w_{1}, w_{2}$, where $L w_{1}=0, L w_{2}=w_{1}$, are given in equation (57), and one finds that

$$
\Psi\left(w_{1}, w_{2}\right)=\alpha_{\star}^{-2}\left(\beta_{0}-\beta_{\star}\right),
$$

where

$$
\begin{aligned}
\Psi\left(\left(\rho_{1}, \zeta_{1}, \phi_{1}, z_{1}\right),\left(\rho_{2}, \zeta_{2}, \phi_{2}, z_{2}\right)\right) & =\left.\Phi^{0}\right|_{0}\left(\left(\rho_{1}, \zeta_{1}, \phi_{1}, z_{1}\right),\left(\rho_{2}, \zeta_{2}, \phi_{2}, z_{2}\right)\right) \\
& =-\beta_{0} a^{-1}(1)\left(\zeta_{2} \rho_{1}-\zeta_{1} \rho_{2}\right)+\int_{0}^{1}\left(z_{2} \phi_{1}-z_{1} \phi_{2}\right) \mathrm{d} s .
\end{aligned}
$$

It follows that $\{e, f\}$, where

$$
e=\alpha_{\star}\left(\beta_{0}-\beta_{\star}\right)^{-1 / 2} w_{1}, \quad f=\alpha_{\star}\left(\beta_{0}-\beta_{\star}\right)^{-1 / 2} w_{2},
$$

is a symplectic basis for the central subspace $X_{1}=P(X)$ of $X$ defined by the spectral projection $P: X \rightarrow X$ corresponding to the purely imaginary part of $L$. The coordinates $q, p$ in the $e$ and $f$ directions are canonical coordinates for $X_{1}$ and the action of the reverser $S$ on this space is given by

$$
S(q, p)=(q,-p)
$$

Modelling the centre manifold $\tilde{X}^{\varepsilon}, \varepsilon=(0, \delta)$ upon the single coordinate chart $\tilde{U}_{1}$ and choosing the coordinate map according to the recipe given in the paragraph below Theorem 3.1, we can identify $\left(\tilde{X}^{\varepsilon}, \tilde{\Phi}^{\varepsilon}, \tilde{H}^{\varepsilon}\right)$ with the two-dimensional canonical Hamiltonian system $\left(\mathcal{M}, \Upsilon, \tilde{H}^{\varepsilon}\right)$, where $\mathcal{M}$ is a neighbourhood of the origin in $\mathbb{R}^{2}$,

$$
\Upsilon\left(\left(q^{1}, p^{1}\right),\left(q^{2}, p^{2}\right)\right)=q^{1} p^{2}-p^{1} q^{2}
$$

and

$$
\tilde{H}^{\varepsilon}(q, p)=K^{\varepsilon}\left(\tilde{u}_{1}+\tilde{r}\left(\tilde{u}_{1} ; \varepsilon\right)\right), \quad \tilde{u}_{1}=q e+p f .
$$

A direct calculation shows that

$$
\tilde{H}_{2}^{0,0}(q, p)=K_{2}^{0,0}\left[\tilde{u}_{1}, \tilde{u}_{1}\right]=\frac{1}{2} p^{2}
$$

where $\varepsilon_{1}^{i} \varepsilon_{2}^{j} \tilde{H}_{k}^{i, j}\left(\tilde{u}_{1}\right)$ denotes the part of the Taylor expansion of $\tilde{H}^{\varepsilon}\left(\tilde{u}_{1}\right)$ which is homogeneous of order $i$ in $\varepsilon_{1}, j$ in $\varepsilon_{2}$ and $k$ in $\tilde{u}_{1} \cong(q, p)$ and $K_{k}^{i, j}$ denotes the symmetric, $k$-linear operator $\mathcal{X}_{1}^{k} \rightarrow$ $\mathbb{R}$ which defines the corresponding coefficient in the Taylor expansion of $K^{\varepsilon}$. Anticipating the scaling $q \sim \delta Q, p \sim \delta^{3 / 2} P$, we write

$$
\tilde{H}^{\varepsilon}(q, p)=\frac{1}{2} p^{2}+c_{1} \delta q^{2}+c_{2} q^{3}+O\left(|p||(\delta, q, p)|^{2}\right)+O\left(|(q, p)|^{2}|(\delta, q, p)|^{2}\right),
$$

so that the first three terms on the right-hand side of the above equation are $O\left(\delta^{3}\right)$ and the remainder is of higher order. The coefficients $c_{1}$ and $c_{2}$ are obtained from the calculations

$$
\begin{aligned}
c_{1} & =K_{2}^{0,1}[e, e]+2 K_{2}^{0,0}\left[e, \tilde{r}_{10}^{0,1}\right] \\
& =K_{2}^{0,1}[e, e]+\Psi\left(L e, \tilde{r}_{10}^{0,1}\right) \\
& =K_{2}^{0,1}[e, e] \\
& =-\frac{1}{2}\left(\beta_{0}-\beta_{\star}\right)^{-1}
\end{aligned}
$$


and

$$
c_{2}=K_{3}^{0,0}[e, e, e]+2 K_{2}^{0,0}\left[e, \tilde{r}_{20}^{0,0}\right]=-\frac{1}{2} c_{0}\left(\beta_{0}-\beta_{\star}\right)^{-3 / 2}, \quad c_{0}=\alpha_{\star}^{3} \int_{0}^{1} a^{-5}(s) \mathrm{d} s,
$$

in which $\tilde{r}_{i j}^{k, \ell}$ denotes the coefficient of $\varepsilon_{1}^{i} \varepsilon_{2}^{j} q^{k} p^{\ell}$ in the Taylor expansion of $\tilde{r}$ and we have made use of the identity

$$
\Psi\left(L \tilde{u}_{1}^{1}, \tilde{u}_{1}^{2}\right)=2 K_{2}^{0,0}\left[\tilde{u}_{1}^{1}, \tilde{u}_{2}^{2}\right] .
$$

Hamilton's equations for $\left(\mathcal{M}, \Upsilon, \tilde{H}^{\varepsilon}\right)$ are

$$
\begin{aligned}
& q_{x}=p+\mathcal{R}_{1}(q, p, \delta) \\
& p_{x}=\delta\left(\beta_{0}-\beta_{\star}\right)^{-1} q+\frac{3}{2} c_{0}\left(\beta_{0}-\beta_{\star}\right)^{-3 / 2} q^{2}+\mathcal{R}_{2}(q, p, \delta),
\end{aligned}
$$

where $\mathcal{R}_{1}, \mathcal{R}_{2}$ are respectively odd and even in their second arguments and

$$
\mathcal{R}_{j}=O(|p||(\delta, q, p)|)+O\left(|(q, p)|^{2}|(\delta, q, p)|\right), \quad j=1,2 .
$$

Introducing the scaled variables

$$
X=\delta^{1 / 2}\left(\beta_{0}-\beta_{\star}\right)^{-1 / 2} x, \quad q(x)=c_{0}^{-1} \delta\left(\beta_{0}-\beta_{\star}\right)^{1 / 2} Q(X), \quad p(x)=c_{0}^{-1} \delta^{3 / 2} P(X),
$$

one finds from (63), (64) that

$$
\begin{aligned}
Q_{X} & =P+\mathcal{R}_{3}(Q, P, \delta), \\
P_{X} & =Q+\frac{3}{2} Q^{2}+\mathcal{R}_{4}(Q, P, \delta),
\end{aligned}
$$

where the remainder terms $\mathcal{R}_{3}$ and $\mathcal{R}_{4}$ are $O\left(\delta^{1 / 2}\right)$ and respectively odd and even in their second arguments. In the limit $\delta \rightarrow 0$ equations (65), (66) are equivalent to

$$
\begin{aligned}
& Q_{X}=P, \\
& P_{X}=Q+\frac{3}{2} Q^{2},
\end{aligned}
$$

whose phase portrait is easily calculated by elementary methods and is depicted in Figure 5. Notice in particular that it has a nonzero equilibrium $(-2 / 3,0)$, surrounded by the symmetric homoclinic orbit

$$
Q(X)=-\operatorname{sech}^{2}\left(\frac{X}{2}\right), \quad P(X)=\operatorname{sech}^{2}\left(\frac{X}{2}\right) \tanh \left(\frac{X}{2}\right) .
$$

One can exploit the reversibility of (65), (66) to deduce that it has a symmetric homoclinic orbit for small positive values of $\delta$. For $\delta=0$ the stable manifold $W_{\mathrm{s}}^{0}$ of the zero equilibrium is known explicitly (it consists of the points on the homoclinic orbit), and since $\left.T W_{\mathrm{s}}^{0}\right|_{(-1,0)}=$ $\{Q=0\}$ it intersects the symmetric section Fix $\mathbf{S}=\{P=0\}$ transversally in the point $(-1,0)$. The stable manifold theorem states that $W_{\mathrm{s}}^{\delta}$ depends uniformly smoothly upon $\delta$, and because the symmetric section is independent of $\delta$ it follows that $W_{\mathrm{s}}^{\delta}$ and Fix S intersect transversally in a 


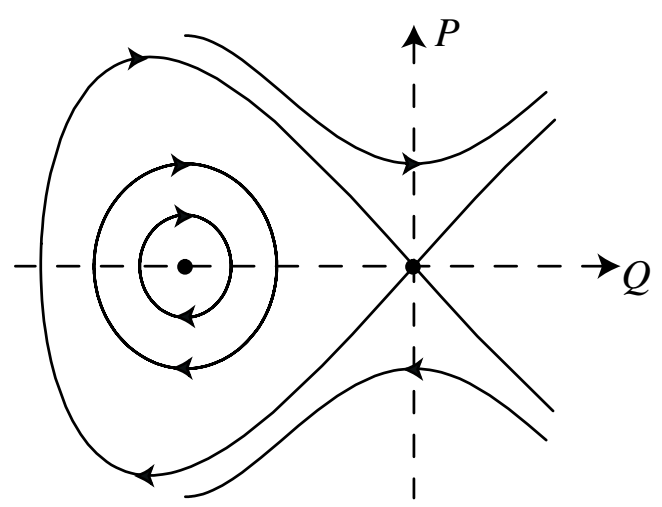

Figure 5: Phase portrait of the scaled reduced system of equations.

point near $(-1,0)$ for sufficiently small positive values of $\delta$. One concludes that the phase portrait of equations (65), (66) has a reversible homoclinic orbit in the left half-plane for sufficiently small positive values of $\delta$.

Tracing back the various changes of variable, one finds that the surface profile of the water corresponding to the homoclinic orbit detected above is given by

$$
\rho(x)=-c_{0}^{-1} \delta \operatorname{sech}^{2}\left(\frac{\delta^{1 / 2} x}{2\left(\beta_{0}-\beta_{\star}\right)^{1 / 2}}\right)+O\left(\delta^{3 / 2}\right) .
$$

We therefore obtain a symmetric solitary wave of depression which decays exponentially and monotonically to a horizontal laminar flow as $x \rightarrow \pm \infty$ and is sketched in Figure 1(b).

\subsection{Homoclinic bifurcation at $C_{1}$}

A Hamiltonian real 1: 1 resonance occurs at points of the curve $C_{1}$ in Figure 4: two pairs of real eigenvalues become complex by colliding at non-zero points on the real axis and forming two Jordan chains of length 2. Of particular interest here is the local part of $C_{1}$ near the point $\left(\beta_{\star}, \alpha_{\star}\right)$, since we can access this curve using the centre-manifold reduction technique with reference value $\left(\beta_{0}, \alpha_{0}\right)=\left(\beta_{\star}, \alpha_{\star}\right)$. We choose values of the bifurcation parameter $\varepsilon=\left(\varepsilon_{1}, \varepsilon_{2}\right)$ in a fashion which enables us to access this curve effectively, namely by writing

$$
\varepsilon_{1}=\beta_{2}(1+\delta) \mu^{2}, \quad \varepsilon_{2}=\alpha_{4} \mu^{4}
$$

where $\beta_{2}, \alpha_{4}$ are the first non-vanishing coefficients in the parameterisation

$$
\beta_{\kappa}=\beta_{\star}+\beta_{2} \kappa^{2}+O\left(\kappa^{4}\right), \quad \alpha_{\kappa}=\alpha_{\star}+\alpha_{4} \kappa^{4}+O\left(\kappa^{6}\right)
$$

of the local part of $C_{1}$ near $\left(\beta_{\star}, \alpha_{\star}\right)$; explicit formulae for $\beta_{2}$ and $\alpha_{4}$ are given in equation (60). Notice that $\mu$ indicates the distance from the point $\left(\beta_{\star}, \alpha_{\star}\right)$, while $\delta$ plays the role of a bifurcation parameter (varying $\delta$ through zero from above we cross the critical curve $C_{1}$ in parameter space from above); the parameter regime marked II in Figure 1(a) corresponds to small, positive values of $\delta$ and $\mu$.

The point $\left(\beta_{0}, \alpha_{0}\right)=\left(\beta_{\star}, \alpha_{\star}\right)$ in parameter space is associated with a Hamiltonian $0^{4}$ resonance ( $L$ has a zero eigenvalue with a Jordan chain of length 4 ); formulae for the generalised 
eigenvectors $w_{j}, j=1, \ldots 4$, where $L w_{1}=0$ and $L w_{j}=w_{j-1}, j=2,3,4$, are given in equations (57)-(59). One finds that

$$
\Psi\left(w_{1}, w_{4}\right)=-d_{1}, \quad \Psi\left(w_{2}, w_{3}\right)=d_{1}, \quad \Psi\left(w_{3}, w_{4}\right)=d_{2}
$$

and the symplectic product of any other combination of these vectors is zero; here $d_{1}>0$ is given by equation (61) and

$$
\begin{aligned}
d_{2}=\beta_{\star}\left(\int_{0}^{1} a^{-3}(s)\right. & \left.\int_{0}^{s} a(t) \int_{0}^{t} a^{-3}(u) \mathrm{d} u \mathrm{~d} t \mathrm{~d} s\right)^{2} \\
& -\int_{0}^{1} a(s)\left(\int_{0}^{s} a^{-3}(t) \int_{0}^{t} a(u) \int_{0}^{u} a^{-3}(v) \mathrm{d} v \mathrm{~d} u \mathrm{~d} t\right)^{2} \mathrm{~d} s .
\end{aligned}
$$

It follows that $\left\{e_{1}, e_{2}, f_{1}, f_{2}\right\}$, where

$$
e_{1}=d_{1}^{-1 / 2}\left(w_{4}+d_{3} w_{2}\right), \quad e_{2}=d_{1}^{-1 / 2} w_{2}, \quad f_{1}=d_{1}^{-1 / 2} w_{1}, \quad f_{2}=d_{1}^{-1 / 2} w_{3}
$$

and $d_{3}=d_{2} / d_{1}$, is a symplectic basis for the central subspace $X_{1}$. The coordinates $q_{1}, q_{2}, p_{1}$ and $p_{2}$ in the $e_{1}, e_{2}, f_{1}$ and $f_{2}$ directions are canonical coordinates for $X_{1}$ and the action of the reverser $S$ on this space is given by

$$
S\left(q_{1}, q_{2}, p_{1}, p_{2}\right)=\left(-q_{1},-q_{2}, p_{1}, p_{2}\right) .
$$

Modelling the centre manifold $\tilde{X}^{\varepsilon}$ upon the single coordinate chart $\tilde{U}_{1}$ and choosing the coordinate map according to the recipe given in the paragraph below Theorem 3.1, we can identify $\left(\tilde{X}^{\varepsilon}, \tilde{\Phi}^{\varepsilon}, \tilde{H}^{\varepsilon}\right)$ with the four-dimensional canonical Hamiltonian system $\left(\mathcal{M}, \Upsilon, \tilde{H}^{\varepsilon}\right)$, where $\mathcal{M}$ is a neighbourhood of the origin in $\mathbb{R}^{4}$,

$$
\Upsilon\left(\left(q_{1}^{1}, q_{2}^{1}, p_{1}^{1}, p_{1}^{1}\right),\left(q_{2}^{2}, q_{2}^{2}, p_{2}^{2}, p_{2}^{2}\right)\right)=q_{1}^{1} p_{1}^{2}+q_{2}^{1} p_{2}^{2}-p_{1}^{1} q_{1}^{2}-p_{2}^{1} q_{2}^{2}
$$

and

$$
\tilde{H}^{\varepsilon}\left(q_{1}, q_{2}, p_{1}, p_{2}\right)=K^{\varepsilon}\left(\tilde{u}_{1}+\tilde{r}\left(\tilde{u}_{1} ; \varepsilon\right)\right), \quad \tilde{u}_{1}=q_{1} e_{1}+q_{2} e_{2}+p_{1} f_{1}+p_{2} f_{2} .
$$

The quadratic part of the reduced Hamiltonian is readily computed; one finds that

$$
\tilde{H}_{2}^{0,0}\left(q_{1}, q_{2}, p_{1}, p_{2}\right)=K_{2}^{0,0}\left[\tilde{u}_{1}, \tilde{u}_{1}\right]=-\frac{d_{3}}{2} q_{1}^{2}-q_{1} q_{2}+\frac{1}{2} p_{2}^{2},
$$

and anticipating the scaling $q_{1} \sim \mu^{7} Q_{1}, q_{2} \sim \mu^{5} Q_{2}, p_{1} \sim \mu^{4} P_{1}, p_{2} \sim \mu^{6} P_{2}$ and the parameterisation (67), we write

$$
\begin{aligned}
& \tilde{H}^{\varepsilon}\left(q_{1}, q_{2}, p_{1}, p_{2}\right)=\frac{1}{2} p_{2}^{2}-q_{1} q_{2} \\
& \quad+c_{1}^{1,0} \varepsilon_{1} p_{1}^{2}+c_{2}^{1,0} \varepsilon_{1} p_{1} p_{2}+c_{6}^{1,0} \varepsilon_{1} q_{2}^{2}+c_{1}^{0,1} \varepsilon_{2} p_{1}^{2}+c_{1}^{2,0} \varepsilon_{1}^{2} p_{1}^{2}+c p_{1}^{3}+\mathcal{R}\left(q_{1}, q_{2}, p_{1}, p_{2}\right)
\end{aligned}
$$

so that the third term on the right-hand side of the above equation is $O\left(\mu^{10}\right)$, the remainder term $\mathcal{R}$ is $O\left(\mu^{14}\right)$ (note that $\tilde{H}^{\varepsilon}\left(-q_{1},-q_{2}, p_{1}, p_{2}\right)=\tilde{H}^{\varepsilon}\left(q_{1}, q_{2}, p_{1}, p_{2}\right)$ becuase of the reversibility) and all other terms are $O\left(\mu^{12}\right)$. 
Proposition 4.1 The Darboux transformation used to construct $\left(\mathcal{M}, \Upsilon, \tilde{H}^{\varepsilon}\right)$ (see equation (35)) can be chosen so that $c_{2}^{1,0}=0$.

Proof. Observe that the change of variable

$$
\bar{q}_{1}=q_{1}-c_{2}^{1,0} \varepsilon_{1} q_{2}, \quad \bar{q}_{2}=q_{2}, \quad \bar{p}_{1}=p_{1}, \quad \bar{p}_{2}=p_{2}+c_{2}^{1,0} \varepsilon_{1} p_{1}
$$

is symplectic and transforms $\left(\mathcal{M}, \Upsilon, \tilde{H}^{\varepsilon}\right)$ into $\left(\mathcal{M}, \Upsilon, \bar{H}^{\varepsilon}\right)$, where

$$
\begin{aligned}
\bar{H}^{\varepsilon}\left(\bar{q}_{1}, \bar{q}_{2}, \bar{p}_{1}, \bar{p}_{2}\right)=\frac{1}{2} \bar{p}_{2}^{2}-\bar{q}_{1} \bar{q}_{2} \\
\quad+c_{1}^{1,0} \varepsilon_{1} \bar{p}_{1}^{2}+\bar{c}_{6}^{1,0} \varepsilon_{1} \bar{q}_{2}^{2}+c_{1}^{0,1} \varepsilon_{2} \bar{p}_{1}^{2}+\bar{c}_{1}^{2,0} \varepsilon_{1}^{2} \bar{p}_{1}^{2}+c \bar{p}_{1}^{3}+\overline{\mathcal{R}}\left(\bar{q}_{1}, \bar{q}_{2}, \bar{p}_{1}, \bar{p}_{2}\right)
\end{aligned}
$$

and $\bar{c}_{6}^{1,0}=c_{6}^{1,0}-c_{2}^{1,0}, \bar{c}_{1}^{2,0}=c_{1}^{2,0}-\frac{1}{2}\left(c_{2}^{1,0}\right)^{2}$ (the remainder term $\overline{\mathcal{R}}$ is $O\left(\mu^{14}\right)$ in the sense explained above). The result follows by replacing the Darboux transformation used in the construction by its composition with the change of variable (69).

To caclulate the remaiing coefficients on the right-hand side of equation (68) we exploit the identity

$$
L \tilde{r}\left(\tilde{u}_{1} ; \varepsilon\right)-\mathrm{d}_{1} \tilde{r}\left[\tilde{u}_{1} ; \varepsilon\right]\left(L \tilde{u}_{1}\right)=-N^{\varepsilon}\left(\tilde{u}_{1}+\tilde{r}\left(\tilde{u}_{1} ; \varepsilon\right)\right)+\mathrm{d}_{1} \tilde{r}\left[\tilde{u}_{1} ; \varepsilon\right]\left(P^{\varepsilon}\left(\tilde{u}_{1}\right)\right)+P^{\varepsilon}\left(\tilde{u}_{1}\right),
$$

is which $P^{\varepsilon}\left(\tilde{u}_{1}\right)$ is the nonlinear part of the reduced Hamiltonian vector field $v_{\tilde{H}^{\varepsilon}}$; this identity is derived by substituting $u=\tilde{u}_{1}+\tilde{r}\left(\tilde{u}_{1} ; \varepsilon\right)$ and $\tilde{u}_{1 x}=L \tilde{u}_{1}+P^{\varepsilon}\left(\tilde{u}_{1}\right)$ into (40)). Let us write

$$
\tilde{H}_{2}^{1,0}\left(q_{1}, q_{2}, p_{1}, p_{2}\right)=c_{1}^{1,0} p_{1}^{2}+c_{3}^{1,0} p_{2}^{2}+c_{4}^{1,0} q_{1}^{2}+c_{5}^{1,0} q_{1} q_{2}+c_{6}^{1,0} q_{2}^{2}
$$

(the coefficients of the remaining terms vanish because of the reversibility), so that

$$
P_{1}^{1,0}\left(\tilde{u}_{1}\right)=2 c_{1}^{1,0} p_{1} e_{1}+2 c_{3}^{1,0} p_{2} e_{2}-\left(2 c_{4}^{1,0} q_{1}+c_{5}^{1,0} q_{2}\right) f_{1}-\left(2 c_{6}^{1,0} q_{2}+c_{5}^{1,0} q_{1}\right) f_{2}
$$

and equating coefficients of $\varepsilon_{1} \tilde{u}_{1}$ on both sides of (70), we find that

$$
\begin{aligned}
& L \tilde{r}_{1000}^{1,0}-\tilde{r}_{0001}^{1,0}-d_{3} \tilde{r}_{0010}^{1,0}=-N_{1}^{1,0}\left[e_{1}\right]-2 c_{4}^{1,0} f_{1}-c_{5}^{1,0} f_{2}, \\
& L \tilde{r}_{0100}^{1,0}-\tilde{r}_{0010}^{1,0}=-N_{1}^{1,0}\left[e_{2}\right]-c_{5}^{1,0} f_{1}-2 c_{6}^{1,0} f_{2}, \\
& L \tilde{r}_{0010}^{1,0}=-N_{1}^{1,0}\left[f_{1}\right]+2 c_{1}^{1,0} e_{1}, \\
& L \tilde{r}_{0001}^{1,0}-\tilde{r}_{0100}^{1,0}=-N_{1}^{1,0}\left[f_{2}\right]+2 c_{3}^{1,0} e_{2} .
\end{aligned}
$$

Here the notation $N_{k}^{i, j}$ and $\tilde{r}_{k}^{i, j}, P_{k}^{i, j}$ is defined analogously to the notation $K_{k}^{i, j}$ and $\tilde{H}_{k}^{i, j}$, while $\tilde{r}_{k_{1} k_{2} k_{3} k_{4}}^{i, j}$ is the coefficient of $\varepsilon_{1}^{i} \varepsilon_{2}^{j} q_{1}^{k_{1}} q_{2}^{k_{2}} p_{1}^{k_{3}} p_{2}^{k_{4}}$ in the Taylor expansion of $\tilde{r}$. Explicit calculations show that $N_{1}^{1,0}\left(e_{1}\right), N_{1}^{1,0}\left(e_{2}\right), N_{1}^{1,0}\left(f_{1}\right)$ vanish while

$$
N_{1}^{1,0}\left(f_{2}\right)=\left(0, d_{1}^{-1 / 2} \beta_{\star}^{-1} \alpha_{\star}^{-1} a(1), 0,0\right) .
$$

It follows by elementary linear algebra that the system of equations (71)-(74) is solvable if and only if

$$
c_{1}^{1,0}=0, \quad c_{6}^{1,0}=-\frac{1}{2 d_{1} \alpha_{\star}^{2}}
$$


and that $\tilde{r}_{0010}^{1,0}=\gamma f_{1}$ for some $\gamma \in \mathbb{R}$.

The value of the coefficient $c_{1}^{0,1}$ is obtained from the calculation

$$
\begin{aligned}
c_{1}^{0,1} & =K_{2}^{0,1}\left[f_{1}, f_{1}\right]+2 K_{2}^{0,0}\left[f_{1}, \tilde{r}_{0010}^{0,1}\right] \\
& =K_{2}^{0,1}\left[f_{1}, f_{1}\right]+\Psi\left(L f_{1}, \tilde{r}_{0010}^{0,1}\right) \\
& =K_{2}^{0,1}\left[f_{1}, f_{1}\right] \\
& =-\frac{1}{2 d_{1} \alpha_{\star}^{2}} .
\end{aligned}
$$

Equating coefficients of $\varepsilon^{2} p_{1}$ on both sides of (70), we find that

$$
L \tilde{r}_{0010}^{2,0}=-N_{1}^{2,0}\left[f_{1}\right]-N_{1}^{1,0}\left[\tilde{r}_{0010}^{1,0}\right]+2 c_{1}^{2,0} e_{1}+c_{2}^{2,0} e_{2},
$$

where the notation for the coefficients in $\tilde{H}_{1}^{0,1}$ is analogous to that used for $\tilde{H}_{1}^{1,0}$. An explicit calculation shows that

$$
N_{1}^{2,0}\left[f_{1}\right]=0, \quad N_{1}^{1,0}\left[\tilde{r}_{0010}^{1,0}\right]=\gamma_{1} N_{1}^{1,0}\left[f_{1}\right]=0,
$$

and it follows from (75) that

$$
c_{1}^{2,0}=\frac{1}{2} \Psi\left(L \tilde{r}_{0010}^{2,0}, f_{1}\right)=-\frac{1}{2} \Psi\left(\tilde{r}_{0010}^{2,0}, L f_{1}\right)=0
$$

(the 'skew-orthogonality' of $L$ with respect to $\Psi$ follows from (62)). Similarly, one finds from the $p_{1}^{3}$ component of (70), namely

$$
L \tilde{r}_{0020}^{0,0}=-N_{2}^{0,0}\left[f_{1}, f_{1}\right]+3 c e_{1},
$$

that

$$
3 c-\Psi\left(N_{2}^{0,0}\left[f_{1}, f_{1}\right], f_{1}\right)=\Psi\left(L \tilde{r}_{2000}^{0,0}, f_{1}\right)=-\Psi\left(\tilde{r}_{2000}^{0,0}, L f_{1}\right)=0
$$

and hence that

$$
c=\frac{1}{3} \Psi\left(N_{2}^{0,0}\left[f_{1}, f_{1}\right], f_{1}\right)=-\frac{1}{2 d_{1}^{3 / 2}} \int_{0}^{1} a^{-5}(s) \mathrm{d} s .
$$

Observe that

$$
c_{6}^{1,0}=-\frac{1}{\beta_{2}}, \quad c_{1}^{0,1}=-\frac{1}{2 \alpha_{4}} .
$$

Choosing $\varepsilon_{1}, \varepsilon_{2}$ according to (67) and introducing the scaled variables

$X=\mu x, \quad q_{1}(x)=\mu^{7} Q_{1}(X), \quad q_{2}(x)=\mu^{5} Q_{2}(X), \quad p_{1}(x)=\mu^{4} P_{1}(X), \quad p_{2}(x)=\mu^{6} P_{2}(X)$, we therefore find that

$$
\tilde{H}^{\varepsilon}\left(q_{1}, q_{2}, p_{1}, p_{2}\right)=\mu^{12}\left[-\frac{1}{2} P_{1}^{2}-(1+\delta) Q_{2}^{2}+\frac{1}{2} P_{2}^{2}-Q_{1} Q_{2}+c P_{1}^{3}\right]+O\left(\mu^{14}\right)
$$

and that Hamilton's equations for $\left(\mathcal{M}, \Upsilon, \tilde{H}^{\varepsilon}\right)$ are

$$
\begin{aligned}
Q_{1 X} & =-P_{1}+3 c P_{1}^{2}+O(\mu), \\
Q_{2 X} & =P_{2}+O(\mu), \\
P_{1 X} & =Q_{2}+O(\mu), \\
P_{2 X} & =2(1+\delta) Q_{2}+Q_{1}+O(\mu) .
\end{aligned}
$$


In the limit $\mu \rightarrow 0$ this dynamical system is equivalent to the single fourth-order ordinary differental equation

$$
\partial_{X}^{4} u-2(1+\delta) \partial_{X}^{2} u+u-u^{2}=0
$$

for the variable $u=3 c P_{1}$.

It was shown by Buffoni, Champneys \& Toland $[6, \S 2]$ that for $\delta=0$ equation (80), and hence the system (76)-(79), has a homoclinic solution which corresponds to a transverse intersection (relative to the zero energy surface) of the stable and unstable manifolds of the zero equilibrium. Since transversality is an open condition, it follows that the same is true of the system (76)(79) for sufficiently small positive values of $\delta$ and $\mu$, for which (76)-(79) is a four-dimensional Hamiltonian system whose linearisation has a plus-minus, complex-conjugate quartet of complex eigenvalues. The work of Devaney [12] therefore implies that there is a Smale horseshoe in the dynamics within the zero energy surface, and implicit in this construction is the existence of infinitely many homoclinic orbits which pass several times through a neighbourhood of the 'primary' transverse homoclinic orbit. These 'multipulse' homoclinic orbits resemble multiple copies of the primary homoclinic orbit, between which there are distributed smaller local maxima and minima, and have an exponentially decaying oscillatory tail at infinity.

Tracing back the various changes of variable, one finds that the surface profile of the water is given by

$$
\rho(x)=d_{1}^{-1 / 2} \mu^{4} P_{1}(\mu x)+O\left(\mu^{5}\right) .
$$

The primary homoclinic orbit $u(x)$ of equation (80) satisfies $\max _{x \in \mathbb{R}} u(x)>0$, so that the homoclinic orbits of (76)-(79) correspond to multi-troughed solitary waves of depression which decay exponentially to a horizontal laminar flow as $x \rightarrow \pm \infty$ (see Figure 1(c)).

\subsection{Homoclinic bifurcation at $C_{2}$}

A Hamiltonian Hopf bifurcation takes place at points of the curve $C_{2}$ in Figure 4: two pairs of purely imaginary eigenvalues become complex by colliding at non-zero points $\pm \mathrm{i} q$ on the imaginary axis and forming two Jordan chains of length 2 . This resonance is associated with the bifurcation of a branch of homoclinic solutions into the region with complex eigenvalues (the parameter regime marked III in Figure 1(a)). Let us therefore fix reference values $\left(\beta_{0}, \alpha_{0}\right) \in C_{2}$ and introduce a bifurcation parameter by choosing $\left(\varepsilon_{1}, \varepsilon_{2}\right)=(0, \delta)$, where $0<\delta \ll 1$.

By normalising $e, f$ and modifying $f$ by the addition of a suitable multiple of $e$ if necessary, we may suppose that the generalised eigenvectors $e, f$, where

$$
L e=\mathrm{i} q e, \quad L \bar{e}=-\mathrm{i} q \bar{e}, \quad(L-\mathrm{i} q I) f=e, \quad(L+\mathrm{i} q I) \bar{f}=\bar{e},
$$

satisfy $S e=\bar{e}, S f=-\bar{f}$ and $\Psi(e, \bar{f})=1, \Psi(f, \bar{e})=-1$ and the symplectic products of all other combinations are zero (note that $\Psi$ acts bilinearly on pairs of complex vectors). It follows that $\{e, f, \bar{e}, \bar{f}\}$ is a symplectic basis for the central subspace $X_{1}$ (so that the coordinates $A, B$, $\bar{A}$ and $\bar{B}$ in the $e, f, \bar{e}$ and $\bar{f}$ directions are canonical coordinates for $X_{1}$ ) and the action of the reverser $S$ on this space is given by

$$
S(A, B)=(\bar{A},-\bar{B}) .
$$

Modelling the centre manifold $\tilde{X}^{\varepsilon}, \varepsilon=(0, \delta)$ upon the single coordinate chart $\tilde{U}_{1}$ and choosing the coordinate map according to the recipe given in the paragraph below Theorem 3.1, we 
can identify $\left(\tilde{X}^{\varepsilon}, \tilde{\Phi}^{\varepsilon}, \tilde{H}^{\varepsilon}\right)$ with the four-dimensional canonical Hamiltonian system $\left(\mathcal{M}, \Upsilon, \tilde{H}^{\varepsilon}\right)$, where $\mathcal{M}$ is a neighbourhood of the origin in $\mathbb{R}^{4}$,

$$
\Upsilon\left(\left(A^{1}, B^{1}, \overline{A^{1}}, \overline{B^{1}}\right),\left(A^{2}, B^{2}, \overline{A^{2}}, \overline{B^{2}}\right)\right)=A^{1} \overline{B^{2}}-A^{2} \overline{B^{1}}+\overline{A^{1}} B^{2}-\overline{A^{2}} B^{1}
$$

and

$$
\tilde{H}^{\varepsilon}(A, B)=K^{\varepsilon}\left(\tilde{u}_{1}+\tilde{r}\left(\tilde{u}_{1} ; \varepsilon\right)\right), \quad \tilde{u}_{1}=A e+B f+\bar{A} \bar{e}+\bar{B} \bar{f} .
$$

The flow of the above four-dimensional Hamiltonian system can be analysed using the theory developed by Iooss \& Pérouème [26] and Buffoni \& Groves [7]. The Birkhoff normal-form theory states that for each $n_{0} \geq 2$ there is a near-identity, analytic, symplectic change of coordinates with the property that

$$
\begin{aligned}
\tilde{H}^{\varepsilon}(A, B)=\mathrm{i} q & (A \bar{B}-\bar{A} B)+|B|^{2} \\
& +H_{\mathrm{NF}}\left(|A|^{2}, \mathrm{i}(A \bar{B}-\bar{A} B), \delta\right)+O\left(|(A, B)|^{2}|(\delta, A, B)|^{n_{0}}\right)
\end{aligned}
$$

in the new coordinates; the function $H_{\mathrm{NF}}$ is a real polynomial of order $n_{0}+1$ which satisfies

$$
H_{\mathrm{NF}}\left(|A|^{2}, \mathrm{i}(A \bar{B}-\bar{A} B), \delta\right)=O\left(|(A, B)|^{2}|(\delta, A, B)|\right),
$$

and in these coordinates Hamilton's equations for the reduced system are given by

$$
\begin{gathered}
A_{x}=\mathrm{i} q A+B+\mathrm{i} A \partial_{2} H_{\mathrm{NF}}\left(|A|^{2}, \mathrm{i}(A \bar{B}-\bar{A} B), \delta\right)+O\left(|(A, B) \|(\delta, A, B)|^{n_{0}}\right), \\
B_{x}=\mathrm{i} q B+\mathrm{i} B \partial_{2} H_{\mathrm{NF}}\left(|A|^{2}, \mathrm{i}(A \bar{B}-\bar{A} B), \delta\right) \\
\quad-A \partial_{1} H_{\mathrm{NF}}\left(|A|^{2}, \mathrm{i}(A \bar{B}-\bar{A} B), \delta\right)+O\left(|(A, B) \|(\delta, A, B)|^{n_{0}}\right) .
\end{gathered}
$$

The theory by Iooss \& Pérouème and Buffoni \& Groves demands that the coefficients $c_{1}$ and $c_{3}$ in the expansion

$$
\begin{aligned}
H_{\mathrm{NF}}=\delta c_{1}|A|^{2}+\delta \mathrm{i} c_{2}(A \bar{B}-\bar{A} B)+c_{3}|A|^{4} \\
+\mathrm{i} c_{4}|A|^{2}(A \bar{B}-\bar{A} B)-c_{5}(A \bar{B}-\bar{A} B)^{2}+\delta^{2} c_{6}|A|^{2}+\delta^{2} \mathrm{i} c_{7}(A \bar{B}-\bar{A} B)+\ldots
\end{aligned}
$$

are respectively negative and positive; the methods explained in Section 4.2 show that

$$
c_{1}=\Psi\left(N_{1}^{0,1}[e], \bar{e}\right)
$$

and

$$
c_{3}=\Psi\left(N_{2}^{0,0}\left[e, \tilde{r}_{1010}^{0,0}\right], \bar{e}\right)+\Psi\left(N_{2}^{0,0}\left[\bar{e}, \tilde{r}_{2000}^{0,0}\right], \bar{e}\right)+\frac{3}{2} \Psi\left(N_{3}^{0,0}[e, e, \bar{e}], \bar{e}\right),
$$

where $\tilde{r}_{1010}^{0,0}$ and $\tilde{r}_{2000}^{0,0}$ are the unique solutions of the equations

$$
\begin{aligned}
L \tilde{r}_{1010}^{0,0} & =-2 N_{2}^{0,0}[e, \bar{e}], \\
(L-2 \mathrm{i} q I) \tilde{r}_{2000}^{, 0} & =-N_{2}^{0,0}[e, e] .
\end{aligned}
$$

Theorem 4.2 Suppose that $c_{1}<0$ and $c_{3}>0$.

(i) Iooss \& Pérouème) For each sufficiently small, positive value of $\delta$ the two-degree-offreedom Hamiltonian system (82), (83) has two distinct symmetric homoclinic solutions. 
(ii) (Buffoni \& Groves) For each sufficiently small, positive value of $\delta$ the two-degree-offreedom Hamiltonian system (82), (83) has an infinite number of geometrically distinct homoclinic solutions which generically resemble multiple copies of one of the homoclinic solutions in part (i).

The homoclinic solutions identified above correspond to envelope solitary waves of amplitude $O\left(\left(-c_{1} \delta\right)^{1 / 2}\right)$ which decay exponentially to a horizontal flow as $x \rightarrow \pm \infty$; they are sketched in Figure 1(c).

Explicit formulae for $c_{1}$ and $c_{3}$ were computed for irrotational waves by Buffoni \& Groves [7, Appendix B], and for general vorticity distributions one can prove that $c_{1}<0, c_{3}>0$ for values of $\left(\beta_{0}, \alpha_{0}\right)$ on the local part of $C_{2}$ near $\left(\beta_{\star}, \alpha_{\star}\right)$. To this end suppose that $\left(\beta_{0}, \alpha_{0}\right)=\left(\beta_{\mu}, \alpha_{\mu}\right)$, where

$$
\beta_{\mu}=\beta_{\star}-2 \alpha_{\star}^{2} d_{1} \mu^{2}+O\left(\mu^{4}\right), \quad \alpha_{\mu}=\alpha_{\star}+\alpha_{\star}^{2} d_{1} \mu^{4}+O\left(\mu^{6}\right),
$$

so that $q=\mu$. We find that

$$
L e_{0}=\mathrm{i} \mu e_{0}, \quad(L-\mathrm{i} \mu I) f_{0}=e_{0},
$$

where

$$
\begin{gathered}
e_{0}=\left(\begin{array}{c}
\mathrm{i} \mu \alpha_{\star}^{-1}+\mathrm{i} \mu^{3} \int_{0}^{1} a^{-3}(s) \int_{0}^{s} a(t) \int_{0}^{t} a^{-3}(u) \mathrm{d} u \mathrm{~d} t \mathrm{~d} s+O_{\mathrm{i}}\left(\mu^{5}\right) \\
\mu^{2} a(1) \alpha_{\star}^{-1}+\mu^{4} a(1) \int_{0}^{1} a^{-3}(s) \int_{0}^{s} a(t) \int_{0}^{t} a^{-3}(u) \mathrm{d} u \mathrm{~d} t \mathrm{~d} s+O_{\mathrm{r}}\left(\mu^{6}\right) \\
\mathrm{i} \mu \int_{0}^{s} a^{-3}(t) \mathrm{d} t+\mathrm{i} \mu^{3} \int_{0}^{s} a^{-3}(t) \int_{0}^{t} a(u) \int_{0}^{u} a^{-3}(v) \mathrm{d} v \mathrm{~d} u \mathrm{~d} t+O_{\mathrm{i}}\left(\mu^{5}\right) \\
\mu^{2} a(s) \int_{0}^{s} a^{-3}(t) \mathrm{d} t+\mu^{4} a(s) \int_{0}^{s} a^{-3}(t) \int_{0}^{t} a(u) \int_{0}^{u} a^{-3}(v) \mathrm{d} v \mathrm{~d} u \mathrm{~d} t+O_{\mathrm{r}}\left(\mu^{6}\right)
\end{array}\right), \\
f_{0}=\left(\begin{array}{c}
\mu^{2} \int_{0}^{1} a^{-3}(s) \int_{0}^{s} a(t) \int_{0}^{t} a^{-3}(u) \mathrm{d} u \mathrm{~d} t \mathrm{~d} s+O_{\mathrm{r}}\left(\mu^{4}\right) \\
-\mathrm{i} \mu a(1) \alpha_{\star}^{-1}-3 \mathrm{i} \mu^{3} a(1) \int_{0}^{1} a^{-3}(s) \int_{0}^{s} a(t) \int_{0}^{t} a^{-3}(u) \mathrm{d} u \mathrm{~d} t \mathrm{~d} s+O_{\mathrm{i}}\left(\mu^{5}\right) \\
2 \mu^{2} \int_{0}^{s} a^{-3}(t) \int_{0}^{t} a(u) \int_{0}^{u} a^{-3}(v) \mathrm{d} v \mathrm{~d} u \mathrm{~d} t+O_{\mathrm{r}}\left(\mu^{4}\right) \\
-\mathrm{i} \mu a(s) \int_{0}^{s} a^{-3}(t) \mathrm{d} t-3 \mathrm{i} \mu^{3} a(1) \int_{0}^{s} a^{-3}(t) \int_{0}^{t} a(u) \int_{0}^{u} a^{-3}(v) \mathrm{d} v \mathrm{~d} u \mathrm{~d} t+O_{\mathrm{i}}\left(\mu^{5}\right)
\end{array}\right)
\end{gathered}
$$

and the symbols $O_{\mathrm{r}}\left(\mu^{n}\right), O_{\mathrm{i}}\left(\mu^{n}\right)$ denote quantities of $O\left(\mu^{n}\right)$ which are respectively real and purely imaginary. Observe that $e_{0}=\mathrm{i} \mu\left(\phi_{\mu}(1),-\mathrm{i} \mu a(1) \phi_{\mu}(1), \phi_{\mu},-\mathrm{i} \mu a(s) \phi_{\mu}\right)^{\mathrm{T}}$, where

$$
T_{\mu} \tilde{\phi}_{\mu}=-\mu^{2} \tilde{\phi}_{\mu}, \quad\left[\tilde{\phi}_{\mu}, \tilde{\phi}_{\mu}\right]_{\mu}=0 \text {. }
$$

The next step is to normalise the generalised eigenvectors. We have that

$$
\Psi\left(e_{0}, \overline{f_{0}}\right)=-\Psi\left(f_{0}, \overline{e_{0}}\right)=d_{4}, \quad \Psi\left(f_{0}, \overline{f_{0}}\right)=\mathrm{i} d_{5},
$$

where

$$
d_{4}=4 d_{1} \mu^{4}+O_{\mathrm{r}}\left(\mu^{6}\right), \quad d_{5}=-4 d_{1} \mu^{3}+O_{\mathrm{r}}\left(\mu^{5}\right),
$$

and the calculations

$$
\Psi\left(e_{0}, \overline{e_{0}}\right)=2 \mathrm{i} \mu^{3}\left[\tilde{\phi}_{\mu}, \tilde{\phi}_{\mu}\right]_{\mu}
$$


and

$$
\mathrm{i} \mu \Psi\left(e_{0}, f_{0}\right)=\Psi\left(L e_{0}, f_{0}\right)=-\Psi\left(e_{0}, L f_{0}\right)=-\Psi\left(e_{0}, \mathrm{i} \mu f_{0}+e_{0}\right)=-\mathrm{i} \mu \Psi\left(e_{0}, f_{0}\right)
$$

imply that $\Psi\left(e_{0}, \bar{e}_{0}\right)=\Psi\left(e_{0}, f_{0}\right)=\Psi\left(\bar{e}_{0}, \bar{f}_{0}\right)=0$. It follows that

$$
e=\frac{\mathrm{i}}{d_{4}^{1 / 2}} e_{0}, \quad f=\frac{\mathrm{i}}{d_{4}^{1 / 2}}\left(f_{0}-\frac{\mathrm{i} d_{5}}{2 d_{4}} e_{0}\right)
$$

satisfy equation (81) and the normalisation requirements given below it.

Performing a series of lengthy calculations, one finds that

$$
c_{1}=\frac{1}{d_{4}} \Psi\left(N_{1}^{0,1}\left[e_{0}\right], \overline{e_{0}}\right)=-\frac{1}{4 d_{1}} \alpha_{\star}^{-2} \mu^{-2}+O_{\mathrm{r}}(1)<0
$$

and

$$
\begin{aligned}
c_{3} & =\frac{1}{d_{4}^{2}} \Psi\left(N_{2}^{0,0}\left[e_{0}, \tilde{R}_{1010}^{0,0}\right], \overline{e_{0}}\right)+\frac{1}{d_{4}^{2}} \Psi\left(N_{2}^{0,0}\left[\overline{e_{0}}, \tilde{R}_{2000}^{0,0}\right], \overline{e_{0}}\right)+\frac{3}{2 d_{4}^{2}} \Psi\left(N_{3}^{0,0}\left[e_{0}, e_{0}, \overline{e_{0}}\right], \overline{e_{0}}\right) \\
& =\frac{19}{64 d_{1}^{3}}\left(\int_{0}^{1} a^{-5}(s) \mathrm{d} s\right)^{2} \mu^{-8}+O_{\mathrm{r}}\left(\mu^{-6}\right)>0,
\end{aligned}
$$

where

$$
\begin{gathered}
\tilde{R}_{1010}^{0,1}=-2 L^{-1} N_{2}^{0,0}\left[e_{0}, \bar{e}_{0}\right]=\left(\begin{array}{c}
-3 d_{1}^{-1} \alpha_{\star}^{-1} \mu^{-2} \int_{0}^{1} a^{-5}(s) \mathrm{d} s+O_{\mathrm{r}}(1) \\
0 \\
-3 d_{1}^{-1} \mu^{-2} \int_{0}^{1} a^{-5}(s) \mathrm{d} s \int_{0}^{s} a^{-3}(t) \mathrm{d} t+O_{\mathrm{r}}(1) \\
0
\end{array}\right), \\
\tilde{R}_{2000}^{0,1}=-(L-2 \mathrm{i} \mu I)^{-1} N_{2}^{0,0}\left[e_{0}, e_{0}\right]=\left(\begin{array}{c}
\frac{1}{6} d_{1}^{-1} \alpha_{\star}^{-1} \mu^{-2} \int_{0}^{1} a^{-5}(s) \mathrm{d} s+O_{\mathrm{r}}(1) \\
-\frac{1}{3} \mathrm{i} d_{1}^{-1} \alpha_{\star}^{-1} \mu^{-1} a(1) \int_{0}^{1} a^{-5}(s) \mathrm{d} s+O_{\mathrm{i}}(\mu) \\
\frac{1}{6} d_{1}^{-1} \mu^{-2} \int_{0}^{1} a^{-5}(s) \mathrm{d} s \int_{0}^{s} a^{-3}(t) \mathrm{d} s+O_{\mathrm{r}}(\mu) \\
-\frac{1}{3} \mathrm{i} d_{1}^{-1} \mu^{-1} a(p) \int_{0}^{1} a^{-5}(s) \mathrm{d} s \int_{0}^{s} a^{-3}(t) \mathrm{d} t+O_{\mathrm{i}}(\mu)
\end{array}\right) .
\end{gathered}
$$

\section{References}

[1] AMick, C. J. \& KIRCHGÄSSnER, K. 1989 A theory of solitary water waves in the presence of surface tension. Arch. Rat. Mech. Anal. 105, 1-49.

[2] Beale, J. T. 1977 The existence of solitary water waves. Commun. Pure Appl. Math. 30, 373-389.

[3] Benjamin, T. B. 1995 Verification of the Benjamin-Lighthill conjecture about steady water waves. J. Fluid Mech. 295, 337-356. 
[4] Binding, P. A., Browne, P. J., Code, W. J. \& Watson, B. A. 2004 Transformation of Sturm-Liouville problems with decreasing affine boundary conditions. Proc. Edin. Math. Soc. 47, 533-552.

[5] BognÁr, J. 1974 Indefinite Inner Product Spaces. New York: Springer-Verlag.

[6] Buffoni, B., Champneys, A. R. \& Toland, J. F. 1996 Bifurcation and coalescence of a plethora of homoclinic orbits for a Hamiltonian system. J. Dyn. Diff. Eqns. 8, 221-279.

[7] Buffoni, B. \& Groves, M. D. 1999 A multiplicity result for solitary gravity-capillary waves in deep water via critical-point theory. Arch. Rat. Mech. Anal. 146, 183-220.

[8] Buffoni, B., Groves, M. D. \& Toland, J. F. 1996 A plethora of solitary gravitycapillary water waves with nearly critical Bond and Froude numbers. Phil. Trans. Roy. Soc. Lond. A 354, 575-607.

[9] Champneys, A. R., Vanden-Broeck, J.-M. \& Lord, G. J. 2002 Do true elevation gravity-capillary solitary waves exist? A numerical investigation. J. Fluid Mech. 454, 403417.

[10] Constantin, A., Sattinger, D. H. \& Strauss, W. 2006 Variational formulations for steady water waves with vorticity. J. Fluid Mech. 548, 151-163.

[11] Constantin, A. \& Strauss, W. 2004 Exact steady periodic water waves with vorticity. Commun. Pure Appl. Math. 57, 481-527.

[12] Devaney, R. L. 1976 Homoclinic orbits in Hamiltonian systems. J. Diff. Eqns. 21, 431438.

[13] Dubreil-Jacotin, M. L. 1934 Sur la détermination rigoureuse des ondes permanentes périodiques d'ampleur finite. J. Math. Pures Appl. 13, 217-291.

[14] Groves, M. D. 1995 A new Hamiltonian formulation of the steady water-wave problem. In Structure and Dynamics of Nonlinear Waves in Fluids (eds. Mielke, A. \& Kirchgässner, K.), pages 259-267. Singapore: World Scientific.

[15] Groves, M. D. 2001 An existence theory for three-dimensional periodic travelling gravity-capillary water waves with bounded transverse profiles. Physica D 152-153, 395415.

[16] Groves, M. D. \& Haragus, M. 2003 A bifurcation theory for three-dimensional oblique travelling gravity-capillary water waves. J. Nonlinear Sci. 13, 397-447.

[17] Groves, M. D., Haragus, M. \& Sun, S.-M. 2002 A dimension-breaking phenomenon in the theory of gravity-capillary water waves. Phil. Trans. Roy. Soc. Lond. A 360, 21892243.

[18] Groves, M. D. \& Mielke, A. 2001 A spatial dynamics approach to three-dimensional gravity-capillary steady water waves. Proc. Roy. Soc. Edin. A 131, 83-136. 
[19] Groves, M. D. \& SAndstede, B. 2004 A plethora of three-dimensional periodic travelling gravity-capillary water waves with multipulse transverse profiles. J. Nonlinear Sci. 14, 297-340.

[20] Groves, M. D. \& Toland, J. F. 1997 On variational formulations for steady water waves. Arch. Rat. Mech. Anal. 137, 203-226.

[21] HuR, V. M. 2005 Exact solitary water waves with vorticity. Preprint. (To appear in SIAM J. Math. Anal.)

[22] Iohvidov, I. S., KReIN, M. G. \& LANGER, H. 1982 Introduction to the Spectral Theory of Operators in Spaces with an Indefinite Metric. Berlin: Akademie-Verlag.

[23] Iooss, G. \& KirchGÄssner, K. 1990 Bifurcation d'ondes solitaires en présence d'une faible tension superficielle. C. R. Acad. Sci. Paris, Sér. 1 311, 265-268.

[24] Iooss, G. \& KIRCHGÄSSnER, K. 1992 Water waves for small surface tension: an approach via normal form. Proc. Roy. Soc. Edin. A 122, 267-299.

[25] Iooss, G. \& KirRmann, P. 1996 Capillary gravity waves on the free surface of an inviscid fluid of infinite depth. Existence of solitary waves. Arch. Rat. Mech. Anal. 136, 1-19.

[26] Iooss, G. \& PÉrouème, M. C. 1993 Perturbed homoclinic solutions in reversible 1:1 resonance vector fields. J. Diff. Eqns. 102, 62-88.

[27] Jones, M. C. W. 1989 Small amplitude gravity-capillary waves in a channel of finite depth. Glasgow Math. J. 31, 141-160.

[28] Keady, G. \& Norbury, J. 1978 On the existence theory for irrotational water waves. Math. Proc. Camb. Phil. Soc. 83, 137-157.

[29] KIRCHGÄSSNER, K. 1988 Nonlinearly resonant surface waves and homoclinic bifurcation. Adv. Appl. Mech. 26, 135-181.

[30] LombARDI, E. 1997 Orbits homoclinic to exponentially small periodic orbits for a class of reversible systems. Application to water waves. Arch. Rat. Mech. Anal. 137, 227-304.

[31] Mielke, A. 1988 Reduction of quasilinear elliptic equations in cylindrical domains with applications. Math. Meth. Appl. Sci. 10, 51-66.

[32] Mielke, A. 1991 Hamiltonian and Lagrangian Flows on Center Manifolds. Berlin: Springer-Verlag.

[33] SACHS, R. L. 1991 On the existence of small amplitude solitary waves with strong surfacetension. J. Diff. Eqns. 90, 31-51.

[34] Sun, S. M. 1999 Nonexistence of truly solitary waves with small surface tension. Proc. Roy. Soc. Lond. A 455, 2191-2228. 
[35] WAHLÉN, E. 2005 Steady periodic capillary-gravity waves with vorticity. Preprint. (To appear in SIAM J. Math. Anal.)

[36] ZEIDLER, E. 1971 Existenzbeweis für cnoidal waves unter Berücksichtigung der Oberflächenspannung. Arch. Rat. Mech. Anal. 41, 81-107.

[37] ZEIDLER, E. 1973 Existenzbeweis für permanente Kapillar-Schwerewellen mit allgemeinen Wirbelverteilungen. Arch. Rat. Mech. Anal. 50, 34-72. 